\title{
MIMO High Frequency Surface Wave Radar Using Sparse Frequency FMCW Signals
}

\author{
Mengguan $\operatorname{Pan}^{1,2}$ and Baixiao Chen ${ }^{1,2}$ \\ ${ }^{1}$ National Laboratory of Radar Signal Processing, Xidian University, Xi'an 710071, China \\ ${ }^{2}$ Collaborative Innovation Center of Information Sensing and Understanding, Xidian University, Xian, China \\ Correspondence should be addressed to Baixiao Chen; bxchen@xidian.edu.cn
}

Received 14 March 2017; Revised 28 May 2017; Accepted 6 June 2017; Published 14 August 2017

Academic Editor: Ana Alejos

Copyright ( 2017 Mengguan Pan and Baixiao Chen. This is an open access article distributed under the Creative Commons Attribution License, which permits unrestricted use, distribution, and reproduction in any medium, provided the original work is properly cited.

\begin{abstract}
The heavily congested radio frequency environment severely limits the signal bandwidth of the high frequency surface wave radar (HFSWR). Based on the concept of multiple-input multiple-output (MIMO) radar, we propose a MIMO sparse frequency HFSWR system to synthesize an equivalent large bandwidth waveform in the congested HF band. The utilized spectrum of the proposed system is discontinuous and irregularly distributed between different transmitting sensors. We investigate the sparse frequency modulated continuous wave (FMCW) signal and the corresponding deramping based receiver and signal processor specially. A general processing framework is presented for the proposed system. The crucial step is the range-azimuth processing and the sparsity of the carrier frequency causes the two-dimensional periodogram to fail when applied here. Therefore, we introduce the iterative adaptive approach (IAA) in the range-azimuth imaging. Based on the initial 1D IAA algorithm, we propose a modified 2D IAA which particularly fits the deramping processing based range-azimuth model. The proposed processing framework for MIMO sparse frequency FMCW HFSWR with the modified 2D IAA applied is shown to have a high resolution and be able to provide an accurate and clear range-azimuth image which benefits the following detection process.
\end{abstract}

\section{Introduction}

High frequency surface wave radar (HFSWR) refers to a classification of radar which operates in the HF band $(3-30 \mathrm{MHz})$ and utilizes the surface wave mode of propagation. HFSWR systems can provide low-cost, 24-hour, all-weather, real-time, over-the-horizon surveillance of large ocean areas in excess of 200 nautical mile exclusive economic zone (EEZ) [1-4]. They can also provide real-time and all-weather measure for the oceanographical parameters including the surface currents, wave spectrum, wind direction, and intensity [5-10]

However, the HF band is a heavily congested part of the radio spectrum which makes it difficult to find a continuous silent frequency band to transmit radar signals. This limits the signal bandwidth of the HF radar system and results in a poor range resolution [11, 12]. In [11], a continuous measurement of noise and interference data in the frequency band of 3-6 MHz at Cape Race, Newfoundland, Canada, in the period between August 1, 1998, and May 10, 2000, shows that channels with a bandwidth of $20 \mathrm{KHz}$ are readily available, while there is no channel available with a bandwidth of $100 \mathrm{KHz}$. Thus a signal bandwidth of several tens of kilohertz is common in typical HFSWR system which corresponds to a range bin of several kilometers.

Discontinuous spectrum signal with separate subbands distributed over a wide spectrum band is a solution to synthesizing a wideband waveform in a highly congested spectrum environment [13-18]. Systems using discontinuous spectrum waveform vary the carrier frequency at specific moments. A synthesizing processing of different segments gives a high resolution range estimate of the targets. In this paper, we combine the multiple-input multiple-output (MIMO) concept with the sparse spectrum signal and convert the temporal frequency diversity to spatial frequency diversity. Since the widespread use of FMCW signal [19] and its variation frequency modulated interrupt continuous wave (FMICW) signal [20] in HFSWR systems, for example, the SeaSonde $[3,9]$ and the WERA [10] systems, we consider the FMCW 
signal specially here and call the proposed system the MIMO sparse frequency FMCW radar system. The resulting system is a multifrequency MIMO radar system, actually, or similar to the synthetic impulse and aperture radar (SIAR) system [21-23]. But different from the conventional multifrequency MIMO radar, whose carrier frequencies are uniformly and continuously distributed, the proposed system has discrete and irregularly distributed spectrum bands. In the meantime, HFSWR systems can benefit from the MIMO concept in several other aspects [24-29]. Firstly, it provides adaptivity on transmit, thus doubling the degrees of freedom (DoF) compared with conventional phased array radar. Therefore, we can put more complexity in the transmitting element and in the limiting case; targets can be located in the horizon plane by using a single trivial receiving element. With the receiver system greatly simplified, it can even be equipped in small vessels [30, 31]. Secondly, MIMO approach has the ability to synthesize virtual antenna positions which results in a larger number of effective array elements. This is of particular interest for HFSWR as large antenna arrays are very costly. Lastly, in MIMO radar systems, orthogonal signals are transmitted by different sensors, resulting in a uniform distribution of the radiation energy in space. This meets the demand of HFSWR system for continuous whole spatial space surveillance.

The key ingredient of MIMO radar operation is that multiple orthogonal waveforms can be used simultaneously. The orthogonality of multichannel sounding signals means that their echoes are allowed to be separated at the receiver. Systems using FMCW or FMICW are quite different from systems using pulsed signal both in receiver structure and following signal processing method as they are deramping processing based, rather than matched filtering based. Naturally, the orthogonality of the waveforms compatible with FMCW radar is different from the counterpart of pulsed radar. MIMO sparse frequency systems with a matched filtering based receiver are presented by Lesturgie and Wang in [28] and [29], respectively. Some orthogonal waveforms for MIMO FMCW radar systems are discussed in [32, 33]. For our proposed MIMO sparse frequency FMCW radar system, the receiver and the signal processor are deramping processing based, and the orthogonality is surely achieved by the frequency offset of different channels.

We establish the signal model of a monostatic MIMO sparse frequency FMCW radar and propose an overall framework for the corresponding receiver and signal processor. We formulate the process of deramping and channel separating. And following crucial step is the range-azimuth processing which synthesizes a wideband signal and a narrow beam associated with the large virtual array. This step is actually the synthetic impulse and aperture processing (SIAP) in conventional SIAR systems $[22,23]$. We set up a twodimensional (2D) spectral analysis model for the rangeazimuth processing. The sparsity of the carrier frequency leads to the irregularity of the sampling instants in the range domain, which results in the high sidelobe levels of the periodogram (matched filtering) output. Therefore different from the continuous spectrum multiple carrier frequency MIMO system, periodogram is not applicable for the sparse frequency MIMO system. In this paper we apply the concept of iterative adaptive approach (IAA) in the rangeazimuth processing. IAA was first proposed in [34] for target direction of arrival (DOA) estimation. It can be interpreted as an iteratively weighted least-square periodogram which eliminates almost completely the leakage problems of the conventional periodogram method in a fully data-adaptive manner [34, 35]. Two-dimensional IAA algorithm has been proposed in [36] for the range-Doppler imaging and in [37] for range-angle processing of MIMO radar. In these papers the sliding window correlating is applied in the range domain which is not applicable in our proposed system as a line spectrum estimation problem is actually incorporated in the range domain. Therefore, a 2D IAA algorithm particularly compatible with our signal model is presented and the complexity is analyzed. And it is verified to be quite suitable as it can provide a clear range-azimuth image with sharp peaks at the locations of the targets and near-zero values at the other locations which benefits the following detection process.

This paper is organized as follows. Section 2 introduces the proposed MIMO sparse frequency FMCW HFSWR system and formulates the signal model. Section 3 presents a general framework for the receiver and signal processor of the proposed system. The input signal to the rangeazimuth processor is formulated and at the end of this section, a two-dimensional spectral analysis model is set up for the range-azimuth processing. Section 4 presents a most intuitive solution, periodogram method for the rangeazimuth processing. The spatial and temporal resolution and the coupling between the two domains are discussed in this section. Section 5 introduces the IAA algorithm into the range-azimuth processing and proposes a two-dimensional IAA algorithm which specially matches our model. Section 6 gives a complete design example for the MIMO sparse frequency FMCW radar. The whole signal processing flow is simulated. The range-azimuth image formed by periodogram and our modified 2D IAA are compared. Finally, conclusions are provided in Section 7.

\section{Signal Model for the MIMO Sparse Frequency FMCW HFSWR System}

A HF radar system using MIMO technique and sparse frequency FMCW signal is illustrated in Figure 1. The number of transmitting sensors is $N_{t}$ and the number of receiving sensors is $N_{r}$. Although the transmitters and receivers are apart, they are close enough to form a monostatic system. In this paper, we set up the model in the case of monostatic. The proposed system is actually a frequency diverse MIMO radar with each transmitting element emitting the same waveform of different carrier frequencies. The carrier frequency of each transmitting element is chosen in the guidance of current spectrum environment. Therefore, a frequency management subsystem should be cooperated to assist the main radar system which is already a common practice for currently deployed HF surface wave or skywave radars [38-41]. The frequency management subsystem monitors the spectrum 


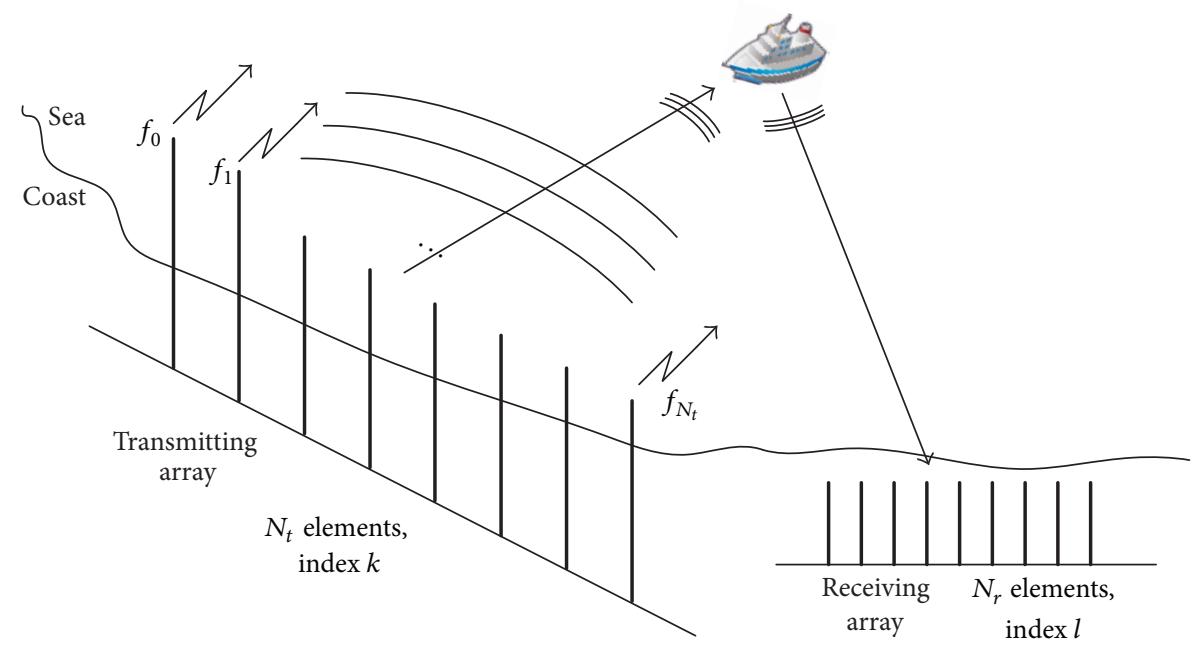

FIgURE 1: A MIMO HFSWR system.

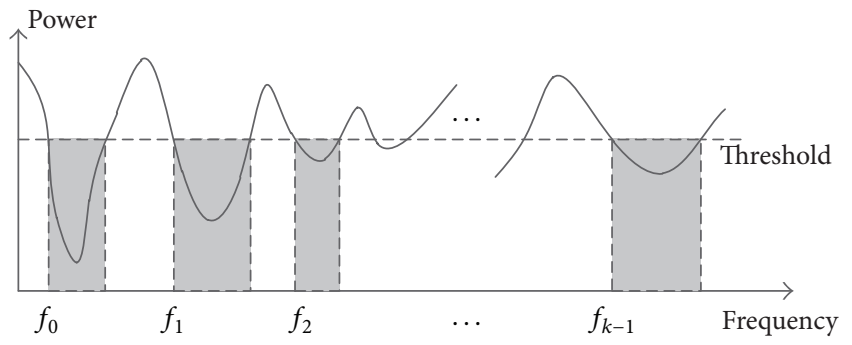

FIGURE 2: HF spectrum distribution illustration.

environment, evaluates the monitoring results, and finally gives suggestions for frequency selection in real-time. It works concurrently with the main radar system. A typical HF spectrum distribution is illustrated in Figure 2. The shading areas below the threshold correspond to the silent frequency bands which can be used by the radar. The $N_{t}$ adjoining relatively silent segments in the frequency span $f_{0}$ to $f_{0}+\Delta F$ are chosen as the working bands of the system. Section 3 presents the requirement of the minimum frequency interval for each transmitted waveform to be separated at the receiver. We denote this frequency interval as $\delta f_{\min }$. Thus in a relatively large silent spectrum block, several working bands can be placed in only if they are at least $\delta f_{\text {min }}$ apart from each other. Let the start frequency and the bandwidth of each segment be $f_{k}$ and $B_{k}, k=0,1, \ldots, N_{t}-1$. The signal bandwidth $B$ shall not exceed the minimum bandwidth of the $N_{t}$ working bands, that is, $B \leq \min _{0 \leq k \leq N_{t}-1}\left\{B_{k}\right\}$. Then all the transmitting elements transmit the same FMCW waveform with bandwidth equal to $B$ and sweep period equal to $T_{m}$ but with different start frequencies $f_{k}$. The timefrequency relationship of the transmitted signals is illustrated in Figure 3.

The transmitted signal of the $k$ th transmitting antenna in a single sweep period is

$$
s_{k}(t)=\widetilde{s}_{k}(t) \cdot \exp \left(j 2 \pi f_{0} t\right), \quad k=0,1, \ldots, N_{t}-1,
$$

where $\widetilde{s}_{k}(t)$ is the baseband waveform and is denoted as

$$
\widetilde{s}_{k}(t)=\operatorname{rect}\left(\frac{t}{T_{m}}\right) \cdot \exp \left(j \pi \mu t^{2}\right) \cdot \exp \left(j 2 \pi \Delta f_{k} t\right)
$$

when using sparse frequency FMCW signal, in which $\operatorname{rect}\left(t / T_{m}\right)=\left\{\begin{array}{l}1,0 \leq t<T_{m} \\ 0,\end{array}\right.$ others is a rectangular window function, $\mu=B / T_{m}$ is the sweep rate, and $\Delta f_{k}=f_{k}-f_{0}$ is the carrier frequency difference between the $k$ th and the first transmitting element. The received signal from a single target is an attenuated, delayed, and Doppler-shifted version of the transmitted signal. In HF radar applications, due to the relatively low speed of ocean surface targets and the relatively large wavelength of HF electromagnetic wave, Doppler frequency of the target is small and is insignificant over a single sweep. Thus the in-sweep Doppler term is omitted [19]. Assuming that there are $Q$ sweep periods in a coherent processing interval (CPI) and only considering the Doppler effect over several sweep periods, the received signal from a moving target in a CPI in $l$ th receiving element after downconverting is given by

$$
\begin{gathered}
x_{l}(t)=\widetilde{\sigma}^{\prime} \cdot\left\{\sum _ { k = 0 } ^ { N _ { t } - 1 } \left[\sum_{q=0}^{Q-1} \widetilde{s}_{k}\left(t-q T_{m}-\tau_{0}\right)\right.\right. \\
\left.\left.\cdot \exp \left(j 2 \pi \frac{2 f_{k} v_{0}}{c} q T_{m}\right)\right] \cdot \exp \left(j 2 \pi f_{0} \tau_{t, k}\right)\right\} \\
\cdot \exp \left(j 2 \pi f_{0} \tau_{r, l}\right), \quad l=0,1, \ldots, N_{r}-1 .
\end{gathered}
$$

Equation (3) is the baseband received signal in the monostatic case where the complex scattering coefficient of the target, which is directly proportional to its corresponding radar cross section (RCS), is represented by $\widetilde{\sigma}, \widetilde{\sigma}^{\prime}$ in (3) equals $\widetilde{\sigma} \exp \left(-j 2 \pi f_{0} \tau_{0}\right), \tau_{0}$ is the round-trip time for the signal to propagate from the array reference point and back, and $v_{0}$ is the radial velocity of the target towards the radar. The terms $\tau_{t, k}$ and $\tau_{r, l}$ refer to the time for a plane wave in the 


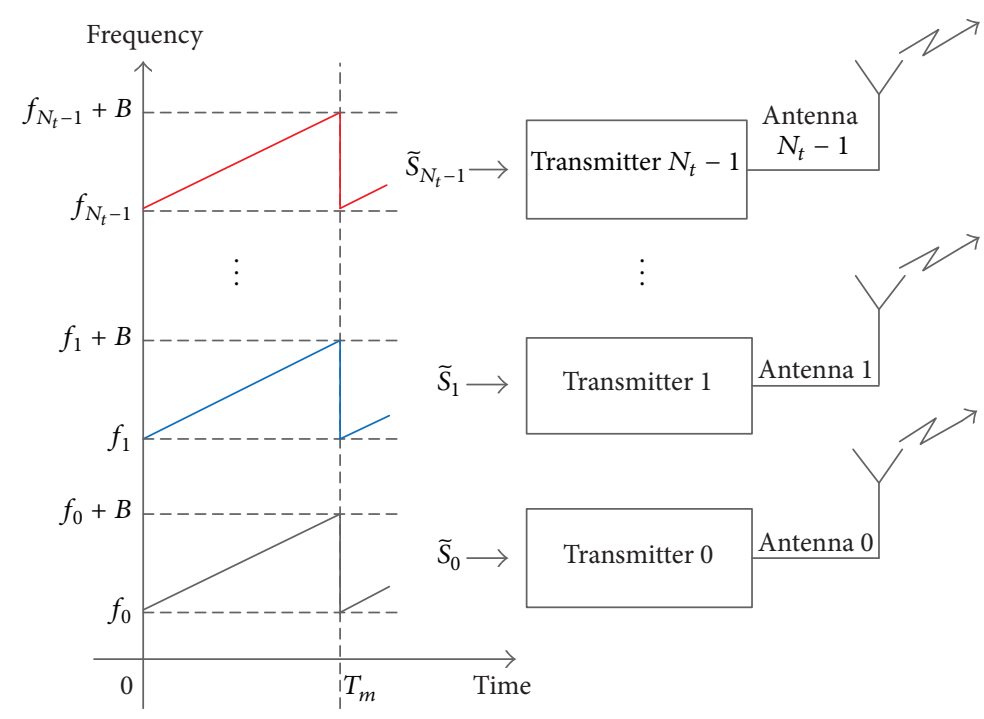

FIGURE 3: Time-frequency characteristic of the transmitted signals.

target direction to travel from the array reference point to the $k$ th transmitting and the $l$ th receiving element, respectively. Equation (3) is deducted under the narrowband assumption and consequently the effect of the travelling time through the array on the complex envelop can be omitted.

Assuming that $\boldsymbol{\eta}_{t, k}$ and $\boldsymbol{\eta}_{r, l}$ are the transmitting and receiving sensor location vectors and $\mathbf{u}$ is the unit vector in the target direction, that is, target direction cosine, then the relative delay of each sensor can be written as

$$
\begin{aligned}
& \tau_{t, k}=\mathbf{u}^{\mathrm{T}} \cdot \frac{\boldsymbol{\eta}_{t, k}}{c}, \quad k=0, \ldots, N_{t}-1, \\
& \tau_{r, l}=\mathbf{u}^{\mathrm{T}} \cdot \frac{\boldsymbol{\eta}_{r, l}}{c}, \quad l=0, \ldots, N_{r}-1,
\end{aligned}
$$

respectively. In this paper, research is done in the horizontal plane (case of HFSWR); thus the location vectors and direction vectors belong to the two-dimensional real space $\mathbb{R}^{2 \times 1}$. Assuming that the target azimuth is $\theta_{0}$, the target direction cosine can be defined as

$$
\mathbf{u}\left(\theta_{0}\right)=\left[\cos \theta_{0}, \sin \theta_{0}\right]^{\mathrm{T}}
$$

where $(\cdot)^{\mathrm{T}}$ represents the transpose operation. Denote the wavenumber vector in the direction of azimuth angle $\theta$ as $\boldsymbol{\kappa}(\theta), \boldsymbol{\kappa}(\theta)=2 \pi \mathbf{u}(\theta) / \lambda$. The phase term related to the target azimuth angle can therefore be written as

$$
\begin{aligned}
& 2 \pi f_{0} \tau_{t, k}=\boldsymbol{\kappa}^{\mathrm{T}}(\theta) \boldsymbol{\eta}_{t, k}, \\
& 2 \pi f_{0} \tau_{r, l}=\boldsymbol{\kappa}^{\mathrm{T}}(\theta) \boldsymbol{\eta}_{r, l} .
\end{aligned}
$$

Based on the upper notation, steering vectors for transmission and reception can be represented in a compact form as

$\mathbf{a}_{t}(\theta)$

$$
=\left[\exp \left(j \boldsymbol{\kappa}^{\mathrm{T}}(\theta) \boldsymbol{\eta}_{t, 0}\right), \ldots, \exp \left(j \boldsymbol{\kappa}^{\mathrm{T}}(\theta) \boldsymbol{\eta}_{t, N_{t}-1}\right)\right]^{\mathrm{T}},
$$

$\mathbf{a}_{r}(\theta)$

$$
=\left[\exp \left(j \boldsymbol{\kappa}^{\mathrm{T}}(\theta) \boldsymbol{\eta}_{r, 0}\right), \ldots, \exp \left(j \boldsymbol{\kappa}^{\mathrm{T}}(\theta) \boldsymbol{\eta}_{r, N_{r}-1}\right)\right]^{\mathrm{T}},
$$

respectively. $\mathbf{a}_{t}(\cdot): \Theta \rightarrow \mathbb{C}^{N_{t} \times 1}$ and $\mathbf{a}_{r}(\cdot): \Theta \rightarrow \mathbb{C}^{N_{r} \times 1}$ are known functions depending on the transmitting and receiving array structure, where $\Theta$ represents the azimuth angle interval of interest. Further, let

$$
\widetilde{\mathbf{s}}(t)=\left[\widetilde{s}_{0}(t), \ldots, \widetilde{s}_{N_{t}-1}(t)\right]^{\mathrm{T}}
$$

consist of all the transmitted signals, and let

$$
\begin{aligned}
& \mathbf{s}_{D}(t) \\
& \quad=\left[\exp \left(j \pi \frac{2 f_{0} v_{0}}{c} t\right), \ldots, \exp \left(j \pi \frac{2 f_{N_{t}-1} v_{0}}{c} t\right)\right]^{\mathrm{T}}
\end{aligned}
$$

consist of Doppler term corresponding to different transmitting channels; (3) can be written in a vector form

$$
\begin{aligned}
\mathbf{x}(t) & =\widetilde{\sigma}^{\prime} \\
\cdot & \left\{\left[\widetilde{\mathbf{s}}\left(t-\left\lfloor\frac{t}{T_{m}}\right\rfloor \cdot T_{m}-\tau_{0}\right) \odot \mathbf{s}_{D}\left(\left\lfloor\frac{t}{T_{m}}\right\rfloor \cdot T_{m}\right)\right]^{\mathrm{T}}\right. \\
\cdot & \left.\mathbf{a}_{t}\left(\theta_{0}\right)\right\} \cdot \mathbf{a}_{r}\left(\theta_{0}\right),
\end{aligned}
$$

where $\odot$ stands for the Hadamard (element-wise) product and $\lfloor\cdot\rfloor$ represents rounded down. A multitarget case can be seen as a superposition of the different echoes at the receiver. 


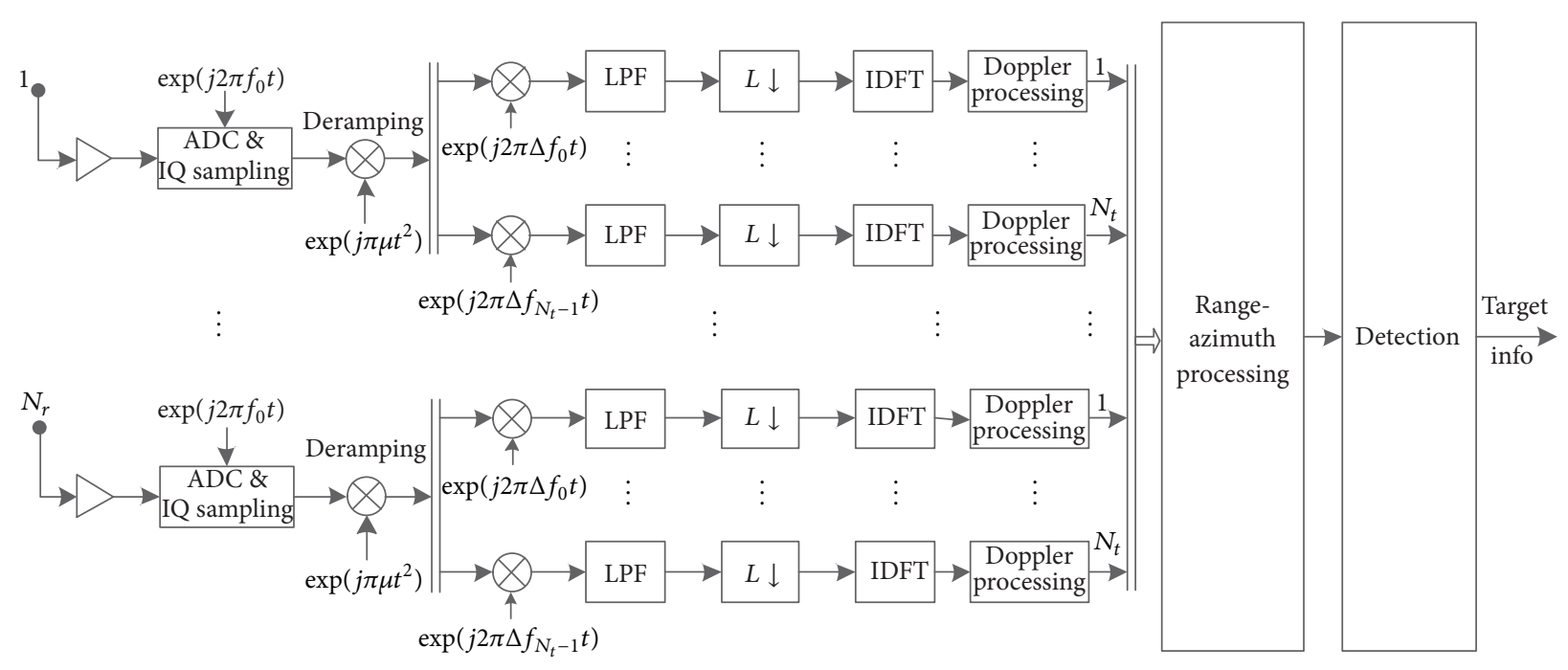

FIGURE 4: Receiver and signal processor structure of the proposed system.

\section{Receiver and Signal Processor Framework for the Proposed System}

A MIMO system using FMCW signal is quite different from the system using pulsed signals both in receiver structure and following signal processing method. It uses stretch processing instead of correlation processing used in pulsed radar to complete the range processing and the channel separating. The receiver and the signal processor structure of the proposed MIMO sparse frequency FMCW radar are shown in Figure 4.

As suggested by Figure 4, the received signal can first be sampled at radio frequency directly and converted to the digital signal. Then digital in-phase quadrature (IQ) sampling is applied to get the baseband IQ signals. The baseband complex signal is mixed with the replica of each baseband transmitted signal. This operation is called the deramping operation and it converts the FM signal to a constantfrequency complex sinusoid which is also known as the beat signal. In the proposed system, output of the $l$ th received signal mixed with the $k$ th transmitted signal is

$$
\begin{gathered}
\tilde{y}_{l, k}(t)=\tilde{\sigma}^{\prime \prime} \cdot \exp \left(j \boldsymbol{\kappa}^{\mathrm{T}} \boldsymbol{\eta}_{r, l}\right) \cdot\left\{\sum_{p=0}^{N_{t}-1} \exp \left(-j 2 \pi \Delta f_{p} \tau_{0}\right)\right. \\
\cdot\left[\sum_{q=0}^{\mathrm{Q}-1} \operatorname{rect}\left(\frac{t-q T_{m}}{T_{m}}\right) \cdot \exp \left(j \boldsymbol{\kappa}^{\mathrm{T}} \boldsymbol{\eta}_{t, p}\right)\right. \\
\cdot \exp \left(j 2 \pi\left(\Delta f_{p}-\Delta f_{k}\right)\left(t-q T_{m}\right)\right) \\
\cdot \exp \left(-j 2 \pi \mu \tau_{0}\left(t-q T_{m}\right)\right) \\
\left.\left.\cdot \exp \left(j 2 \pi \frac{2 f_{p} v_{0}}{c} q T_{m}\right)\right]\right\}, \\
l=0, \ldots, N_{r}-1, k=0, \ldots, N_{t}-1
\end{gathered}
$$

in which $\widetilde{\sigma}^{\prime \prime}=\widetilde{\sigma}^{\prime} \cdot \exp \left(j \pi \mu \tau_{0}^{2}\right)$. And note that the effect of the target delay on the rectangular envelop is omitted since in HF radar applications the maximum potential target delay usually satisfies $\tau_{\max } \ll T_{m}$ [19]. As shown in (12), $\tilde{y}_{l, k}$ is composed by the harmonic components:

$$
\begin{aligned}
\exp \left(-j 2 \pi \mu \tau_{0} t\right) \cdot \exp \left(j 2 \pi\left(\Delta f_{p}-\Delta f_{k}\right) t\right) & \\
& p=0, \ldots, N_{t}-1,
\end{aligned}
$$

and $\exp \left(-j 2 \pi \mu \tau_{0} t\right)$ is the desired one. Let

$$
\delta f_{\min }=\min \left(\Delta f_{k}-\Delta f_{l}\right), \quad k, l=0, \ldots, N_{t}-1, k \neq l
$$

represent the minimum frequency interval between different transmitted signals. To prevent the aliasing of different channels in the frequency domain, $\delta f_{\text {min }}$ should satisfy

$$
\delta f_{\min }>\mu \tau_{\max }
$$

And then to effectively isolate different transmitting channels, the following low-pass filter (LPF) should reserve all the frequency components ranging from 0 to $\mu \tau_{\max }$ and meanwhile prevent any components larger than $\delta f_{\min }$. This low-pass filter is also known as the channel separation filter (CSF). The lowpass filter output of the $(l, k)$ th channel is

$$
\begin{aligned}
y_{l, k}(t)= & \widetilde{\sigma}^{\prime \prime} \cdot \exp \left(j \boldsymbol{\kappa}^{\mathrm{T}}\left(\boldsymbol{\eta}_{r, l}+\boldsymbol{\eta}_{t, k}\right)\right) \\
& \cdot \exp \left(-j 2 \pi \Delta f_{k} \tau_{0}\right) \cdot \sum_{q=0}^{\mathrm{Q}-1} \operatorname{rect}\left(\frac{t-q T_{m}}{T_{m}}\right) \\
& \cdot \exp \left(-j 2 \pi \mu \tau_{0}\left(t-q T_{m}\right)\right) \\
& \cdot \exp \left(j 2 \pi \frac{2 f_{k} v_{0}}{c} q T_{m}\right), \\
& \quad l=0, \ldots, N_{r}-1, k=0, \ldots, N_{t}-1 .
\end{aligned}
$$


Due to the significant bandwidth decrease after deramping and low-pass filtering, the data rate can also be decreased. Thus a decimation routine is followed to lower the data rate and further reduce the computational burden for the succeeding processing flow. The decimation ratio $L$ can be determined by

$$
L=\left\lfloor\frac{F_{s}}{\mu \tau_{\max }}\right\rfloor,
$$

where $F_{s}$ is the original sampling rate of the baseband IQ signals.

After channel separating, as shown in Figure 4, range transforming and Doppler processing are followed in each of the $N_{t} N_{r}$ data streams. Range transforming is actually a spectral analysis procedure and is used to extract the range information. Doppler processing is also referred to as coherent integration and it is essentially crucial in HF radar applications for the following two accounts. Firstly, in the HF band, noise component is dominated by the external noise, which comes from a wide range of sources, such as atmospheric, galactic, and man-made noise [42]. Therefore, the input signal-to-noise ratio (SNR) is quite low and thus a long coherent integration time is required to lift the returns of the targets above that of the noncoherent background noise. In typical HF radar applications for target detection, the integration time is usually several minutes. On the other hand, Doppler processing is required to separate targets and the strong sea clutter in the Doppler domain.

Similar to the conventional FMCW radar, range-Doppler processing usually incorporates two sets of discrete Fourier transforms (DFTs or inverse discrete Fourier transforms (IDFTs)). The first set is done within each sweep period to give range information while the second set is done over several sweep periods in each range bin to give velocity information. Different from the conventional single-frequency FMCW radar, due to the hopping of the carrier frequencies at different transmitting channels of the sparse frequency MIMO HF radar, a single target's velocity corresponds to different Doppler frequencies. Thus the velocity of the Doppler processor output in $(l, k)$ th channel should be calibrated according to the $k$ th carrier frequency $f_{k}$. For the grid point on the range-velocity plane with frequency in range domain equal to $\mu \tau_{0}$ and Doppler frequency equal to $2 f_{k} v_{0} / c$, phase terms $-2 \pi \mu \tau_{0}\left(t-q T_{m}\right)$ and $2 \pi\left(2 f_{k} \nu_{0} / c\right) q T_{m}$ are compensated and a peak emerges at the same location in the rangevelocity image of all the $N_{t} N_{r}$ data streams. The targets' coarse ranges and velocities can then be determined from the rangevelocity image.

Values at the same coarse range cell and the same velocity cell are each collected into a $N_{t} \times N_{r}$ data matrix. Each data matrix corresponds to a grid point in the range-Doppler plane. The following range-azimuth processing should be applied to each grid point within a range and Doppler window to further determine the target azimuth angles and resolve the targets falling in the same range-velocity cell.
For the data matrix at the grid point of the target, which is denoted as $\mathbf{Y}$, its elements are

$$
\begin{aligned}
& Y_{l, k}= \tilde{\sigma}^{\prime \prime \prime} \cdot \exp \left(j \boldsymbol{\kappa}^{\mathrm{T}}\left(\theta_{0}\right) \boldsymbol{\eta}_{r, l}\right) \cdot \exp \left(-j 2 \pi \Delta f_{k} \tau_{0}\right) \\
& \cdot \exp \left(j \boldsymbol{\kappa}^{\mathrm{T}}\left(\theta_{0}\right) \boldsymbol{\eta}_{t, k}\right), \\
& \quad l=0, \ldots, N_{r}-1, k=0, \ldots, N_{t}-1,
\end{aligned}
$$

where $\widetilde{\sigma}^{\prime \prime \prime}$ incorporates the gain of range-velocity processing. Assuming that there are $P$ sampling points in each sweep period after the decimating, we have $\widetilde{\sigma}^{\prime \prime \prime}=P Q \cdot \widetilde{\sigma}^{\prime \prime}$.

Let

$$
\mathbf{a}_{\tau}(\tau)=\left[\exp \left(-j 2 \pi \Delta f_{0} \tau\right), \ldots, \exp \left(-j 2 \pi \Delta f_{N_{t}-1} \tau\right)\right]^{\mathrm{T}}
$$

represent the steering vector relating to the target delay. $\mathbf{a}_{\tau}(\cdot)$ : $T \rightarrow \mathbb{C}^{N_{t} \times 1}$ is a known function depending on the carry frequency distribution, where $T$ represents the target delay interval of interest. Note that the target delay interval of interest that makes sense should be within current coarse range cell. Therefore, the equivalent transmitting steering vector which depends on both range and azimuth is

$$
\widetilde{\mathbf{a}}_{t}(\tau, \theta)=\mathbf{a}_{t}(\theta) \odot \mathbf{a}_{\tau}(\tau) .
$$

Thus the data matrix $\mathbf{Y}$ can be expressed as

$$
\mathbf{Y}=\tilde{\sigma}^{\prime \prime \prime} \cdot \widetilde{\mathbf{a}}_{t}\left(\tau_{0}, \theta_{0}\right) \cdot \mathbf{a}_{r}^{\mathrm{T}}\left(\theta_{0}\right)
$$

The data matrix Y can be vectorized by stacking the columns which yields a $N_{t} N_{r} \times 1$ vector

$$
\dot{\mathbf{y}}=\tilde{\sigma}^{\prime \prime \prime} \cdot \mathbf{a}_{r}\left(\theta_{0}\right) \otimes \widetilde{\mathbf{a}}_{t}\left(\tau_{0}, \theta_{0}\right)=\widetilde{\sigma}^{\prime \prime} \cdot \mathbf{a}\left(\tau_{0}, \theta_{0}\right),
$$

where $\mathbf{a}(\tau, \theta)=\mathbf{a}_{r}(\theta) \otimes \widetilde{\mathbf{a}}_{t}(\tau, \theta)$ is a $N_{t} N_{r} \times 1$ steering vector associated with a virtual array of $N_{t} N_{r}$ sensors.

Considering the multitarget situation, the input data vector of the range-azimuth processor is

$$
\stackrel{\mathbf{y}}{=} \sum_{i=1}^{I} \widetilde{\sigma}_{i}^{\prime \prime \prime} \cdot \mathbf{a}\left(\widetilde{\tau}_{i}, \widetilde{\theta}_{i}\right)+\mathbf{n},
$$

where $\mathbf{n} \in \mathbb{C}^{N_{t} N_{r} \times 1}$ is a noise term, $I$ is the unknown number of targets, $\widetilde{\tau}_{i}$ and $\tilde{\theta}_{i}$ represent the delay and azimuth angle of the $i$ th target, respectively. Equation (23) is a common model of two-dimensional spectral analysis where $\mathbf{y}$ represents the measurement vector and $\mathbf{a}(\cdot): \Omega \rightarrow \mathbb{C}^{N_{t} N_{r} \times 1}$ is a known function determined by both the array structure and the signal structure where $\Omega=\{(\tau, \theta) \mid \tau \in T, \theta \in \Theta\}$ represents the parameter set including all the parameter combinations of interest.

To assist in explaining the solution to the twodimensional spectral analysis problem, the model of (23) is extended. We use a two-dimensional grid to cover $\Omega$; that is, a uniform grid $\left\{\tau_{n}\right\}_{n=1}^{N}$ covers $T$ and a uniform grid $\left\{\theta_{m}\right\}_{m=1}^{M}$ covers $\Theta$. When the grid is fine enough, the parameter estimates of off-grid targets are sufficiently accurate. Thus we assume that $\left\{\left(\widetilde{\tau}_{i}, \widetilde{\theta}_{i}\right)\right\}_{i=1}^{I}$ lie on (practically, 
close to) the grid. This means that there exist $n_{1}, \ldots, n_{I}$ and $m_{1}, \ldots, m_{I}$ such that $\widetilde{\tau}_{i}=\tau_{n_{i}}, \widetilde{\theta}_{i}=\theta_{m_{i}}, i=1, \ldots, I$. Also let

$$
\begin{aligned}
& \mathbf{a}_{n, m}=\mathbf{a}\left(\tau_{n}, \theta_{m}\right), \quad n=1, \ldots, N, m=1, \ldots, M, \\
& \sigma_{n, m}= \begin{cases}\widetilde{\sigma}_{i}^{\prime \prime \prime}, & n=n_{i}, \quad m=m_{i} \\
0, & \text { elsewhere. }\end{cases}
\end{aligned}
$$

Using this notation, (23) can be rewritten as

$$
\stackrel{\circ}{\mathbf{y}}=\sum_{n=1}^{N} \sum_{m=1}^{M} \sigma_{n, m} \mathbf{a}_{n, m}+\mathbf{n} .
$$

The problem of interest in the range-azimuth processing, then, is to accurately estimate the reflection coefficient $\sigma_{n, m}$ on each grid point of the range-azimuth plane from the measurement vector $\dot{\mathbf{y}}_{\mathbf{y}}$ and vectors $\mathbf{a}_{n, m}$ which are already known given the array structure and the waveform parameters.

A detection routine is followed to extract the target information including the target range, azimuth angle, and velocity. The frequency sparsity of the transmit waveform of our proposed system usually leads to the high sidelobe levels of the range-azimuth image, confusing the following detection process. Thus applying a proper range-azimuth processing algorithm; that is, a proper $2 \mathrm{D}$ spectral analysis algorithm in this system is crucial. And we will focus on the range-azimuth processing in the following sections.

\section{Range-Azimuth Processing Using Periodogram}

The most intuitive solution to the $2 \mathrm{D}$ spectral analysis problem represented by (25) is the classical Fourier transformbased periodogram (FP), which is also known as matched filtering (MF) method or single-frequency least-square (SFLS) method. The filter coefficients used match the received signal structure, which yields an optimum output signal-to-noise ratio (SNR).

Periodogram estimates $\sigma_{n, m}$ in a one-by-one manner, simply ignoring the presence of other possible signal components. The estimation of $\sigma_{n, m}$ of current interest is the solution to the following least-squares (LS) data fitting problem:

$$
\widehat{\sigma}_{n, m}=\arg \min _{\sigma_{n, m}}\left\|\stackrel{\circ}{\mathbf{y}}-\sigma_{n, m} \mathbf{a}_{n, m}\right\|^{2} .
$$

The solution to (26) results in a range-azimuth spectrum

$$
\sigma_{n, m}=\frac{\mathbf{a}_{n, m}^{H} \dot{\mathbf{y}}}{\left\|\mathbf{a}_{n, m}\right\|^{2}}, \quad n=1, \ldots, N, m=1, \ldots, M .
$$

The periodogram method represented by (27) is in fact a $2 \mathrm{D}$ range-azimuth matched filtering. It is the method applied in the real-time system of conventional continuous spectrum multiple carrier frequency MIMO radar (or SIAR) [21, 22, 30]. We have extended it to the case of sparse frequency MIMO radar by generalizing the steering vector. And we can call it the conventional approach. In a single target situation, the output is the ambiguity function of the system which reveals the temporal and spatial resolution and the coupling of the two domains. We write below the continuous form instead of the sampled form shown in (27) for the sake of simplification of the analysis:

$$
\begin{aligned}
& \sigma(\tau, \theta)=\frac{1}{N_{t} N_{r}} \mathbf{a}^{H}(\tau, \theta)\left(\tilde{\sigma}_{0} \cdot \mathbf{a}\left(\tau_{0}, \theta_{0}\right)\right)=\frac{\widetilde{\sigma}_{0}}{N_{t} N_{r}} \\
& \cdot \sum_{l=0}^{N_{r}-1} \sum_{k=0}^{N_{t}-1} \exp \left(-j\left(\boldsymbol{\kappa}(\theta)-\boldsymbol{\kappa}\left(\theta_{0}\right)\right)\left(\boldsymbol{\eta}_{r, l}+\boldsymbol{\eta}_{t, k}\right)\right) \\
& \cdot \exp \left(j 2 \pi \Delta f_{k}\left(\tau-\tau_{0}\right)\right) .
\end{aligned}
$$

Assuming that the target delay has been well compensated, we get the zero-delay cut of the ambiguity function:

$$
\begin{aligned}
& \sigma\left(\tau_{0}, \theta\right)=\frac{\widetilde{\sigma}_{0}}{N_{t} N_{r}} \\
& \quad \cdot \sum_{l=0}^{N_{r}-1} \sum_{k=0}^{N_{t}-1} \exp \left(-j\left(\boldsymbol{\kappa}(\theta)-\boldsymbol{\kappa}\left(\theta_{0}\right)\right)\left(\boldsymbol{\eta}_{r, l}+\boldsymbol{\eta}_{t, k}\right)\right) \\
& =\frac{\widetilde{\sigma}_{0}}{N_{t} N_{r}} \mathbf{a}^{H}\left(\tau_{0}, \theta\right) \cdot \mathbf{a}\left(\tau_{0}, \theta_{0}\right),
\end{aligned}
$$

which is actually the beam pattern of a virtual array of $N_{t} N_{r}$ sensors with each element located at $\left(\boldsymbol{\eta}_{r, l}+\boldsymbol{\eta}_{t, k}\right), l=$ $0, \ldots, N_{r}-1, k=0, \ldots, N_{t}-1$. This is also known as the aperture extension property of the MIMO radar [26]. Thus a larger equivalent antenna aperture is obtained, which leads to a higher angular resolution.

Similarly, setting $\theta=\theta_{0}$ in (28) gives the zero-azimuth cut of the ambiguity function:

$$
\sigma\left(\tau, \theta_{0}\right)=\frac{\widetilde{\sigma}_{0}}{N_{t}} \sum_{k=0}^{N_{t}-1} \exp \left(j 2 \pi \Delta f_{k}\left(\tau-\tau_{0}\right)\right) .
$$

Equation (30) is equivalent to an IDFT operation applied to a sequence sampled from a frequency domain signal $\exp (j 2 \pi f \tau)$ at nonuniform distributed frequency points $\Delta f_{k}$, $k=0, \ldots, N_{t}-1$. Thus the resolution in the time domain is proportional to the reciprocal of the whole frequency span $\Delta F$. Then resolution of the fine measurement of the target is

$$
\Delta R=\frac{c}{2 \Delta F}
$$

Therefore the fine range resolution solely depends on the total frequency span $(\Delta F)$ of all the transmitted signals. Even in a congested radio spectrum environment, we can adopt the waveform in an appropriate frequency span with each segment filled in the clean part of the spectrum to obtain the desired range resolution.

The operation represented by (30) is quite similar to the IDFT coherent synthesis method for the stepped frequency signals to obtain high range resolution profile (HRRP) of the targets. In this situation, the stepping of the temporal frequency is converted to the diversity of the spatial frequency. 
Actually, the frequency diverse MIMO radar is also known as synthetic impulse and aperture radar (SIAR) in early literature $[21,23]$. The $2 \mathrm{D}$ range-azimuth periodogram (matched filtering) synthesizes a large virtual aperture composed by both the transmitting and receiving sensors and a much narrower pulse. Thus a much better resolution in both azimuth and range domains is obtained.

While in conventional continuous spectrum SIAR systems, $2 \mathrm{D}$ periodogram approach is nearly sufficient to obtain the desired performance, the spectra discontinuity between signals of different transmitting sensors in the proposed MIMO sparse frequency FMCW system leads to the high sidelobe levels of the periodogram output which is totally unacceptable. Thus a more elaborate method is motivated to be applied here to solve the two-dimensional spectral analysis problem of (25).

When considering the coupling between the range and azimuth angle domain, the receiving array is unconcerned. It can even be discussed by assuming that only a single sensor is used for receiving. Let $N_{r}=1$ in (28); we get the transmitting ambiguity function which can be written as

$$
\begin{aligned}
\sigma_{t}(\tau, \theta)= & \frac{1}{N_{t}} \widetilde{\mathbf{a}}_{t}^{H}(\tau, \theta)\left(\widetilde{\sigma}_{0} \cdot \widetilde{\mathbf{a}}_{t}\left(\tau_{0}, \theta_{0}\right)\right) \\
= & \frac{\widetilde{\sigma}_{0}}{N_{t}} \sum_{k=0}^{N_{t}-1} \exp \left(-j\left(\boldsymbol{\kappa}(\theta)-\boldsymbol{\kappa}\left(\theta_{0}\right)\right) \boldsymbol{\eta}_{t, k}\right) \\
& \cdot \exp \left(j 2 \pi \Delta f_{k}\left(\tau-\tau_{0}\right)\right) .
\end{aligned}
$$

When the transmitting array is a ULA with interelement spacing equal to $d_{t}$, the transmitting ambiguity function is

$$
\begin{aligned}
\sigma_{t}(\tau, \theta)= & \frac{\widetilde{\sigma}_{0}}{N_{t}} \sum_{k=0}^{N_{t}-1} \exp \left(-j \frac{2 \pi}{\lambda} k d_{t}\left(\sin \theta-\sin \theta_{0}\right)\right) \\
& \cdot \exp \left(j 2 \pi \Delta f_{k}\left(\tau-\tau_{0}\right)\right)
\end{aligned}
$$

The coupling between range and azimuth angle mean that, due to an inaccurate direction measurement, the target direction is mismatched, which will shift the target peak location in the range domain and thus influence the measurement of the target range; in the same way, an inaccurate range measurement will influence the measurement of the target azimuth angle. The range-azimuth coupling phenomenon is evident from the intensity plot or the contour plot of the range-azimuth ambiguity function. When coupling occurs, there exists a skewed ridge at the peak location of the ambiguity function while when the coupling is eliminated, the mainlobe of the ambiguity function is distributed symmetrically relative to range and azimuth axes. Letting $\tau=$ $\tau_{0}+\Delta \tau, \theta=\theta_{0}+\Delta \theta$, nearby the location of the target, (33) can be approximated as

$$
\begin{aligned}
\sigma_{t}(\tau, \theta)= & \frac{\widetilde{\sigma}_{0}}{N_{t}} \sum_{k=0}^{N_{t}-1} \exp \left(-j \frac{2 \pi}{\lambda} k d \cos \theta_{0} \Delta \theta\right) \\
& \cdot \exp \left(j 2 \pi \Delta f_{k} \Delta \tau\right) .
\end{aligned}
$$

When the phase terms $(2 \pi / \lambda) k d \cos \theta_{0} \Delta \theta$ and $2 \pi \Delta f_{k} \Delta \tau$ are identical for $k=0, \ldots, N_{t}-1$, peaks will emerge at a skewed ridge which is away from the target location. When the carrier frequencies of the transmitting signals are sequentially distributed, this phenomenon is more likely to occur since the phase terms $(2 \pi / \lambda) k d \cos \theta_{0} \Delta \theta$ and $2 \pi \Delta f_{k} \Delta \tau$ are both ascending in this situation. Thus the range-azimuth coupling is serious when using sequential carrier frequency distribution.

Eliminating the range-azimuth coupling means eliminating the shift of the peak location in the range or azimuth domain due to the bias in the other domain. A simple but effective method is to rearrange the carrier frequencies of the signals radiated from different transmit antennas. The routine of searching for the best arrangement of the carrier frequencies over the transmitting sensors can be called the transmitting frequency distribution optimization. The corresponding object function can be constructed as

$$
F(\mathbf{f})=\left|\theta_{0}-\max _{\theta}\left\{\sigma_{t}\left(\tau_{0}+\Delta \tau, \theta\right)\right\}\right|
$$

or

$$
F(\mathbf{f})=\left|\tau_{0}-\max _{\tau}\left\{\sigma_{t}\left(\tau, \theta_{0}+\Delta \theta\right)\right\}\right|,
$$

in which the vector $\mathbf{f}$ represents the distribution of the carrier frequencies. The entries of $\mathbf{f}$ belong to the initial carrier frequency set which is denoted as $J . \Delta \tau$ and $\Delta \theta$ in (35) and (36) shall not exceed half of the minimum resolution cell. Thus the transmitting frequency distribution optimization problem can be formulated as

$$
\mathbf{f}=\min _{f_{k} \in J}\{F(\mathbf{f})\}
$$

It is actually a combinatorial optimization problem and can be properly solved by several random searching algorithms such as the genetic algorithm.

References [22, 31] also analyze the range-angle coupling of the SIAR system and optimize the carrier frequency distribution from the viewpoint of Fisher information matrix, which can also be applied here.

\section{Range-Azimuth Processing Using Two-Dimensional IAA Method}

Periodogram uses solution to the LS fitting problem (see (26)) as the estimate in each grid point of the range-azimuth plane. It is a data-independent approach. While estimating a spectrum value of a specific grid point $\left(\tau_{n}, \theta_{m}\right)$, the other components located at points different from the current one perform as interferences which will cause the high sidelobe levels of the output spectrum. A prewhitening of the other signal components except the current processing one can cancel strong interferences in the data. The IAA algorithm is inspired by this concept and it uses a weighted least-square (WLS) fitting criterion with the weight matrix equal to the inversion of the interferences and noise covariance matrix. Thus the prewhitening process is actually included. 
The problem of interest in the range-azimuth processing of our proposed system is represented by (25), which is actually a two-dimensional spectral analysis problem with a single snapshot. References $[36,37]$ have already extended the original IAA method to range-Doppler imaging and rangeangle processing in MIMO radar. However, just as there are distinct differences between the matched filtering structure and the deramping processing structure, the proposed deramping based model is also different from the previous $2 \mathrm{D}$ model especially in the range domain. Thus in this section we present a concise modified 2D IAA solution which is specially compatible with our proposed model.

In fact, assuming that a single subscript $\zeta$ takes the place of the two-dimensional subscript $(m, n)$ such that

$$
\zeta=n+(m-1) N, \quad n=1, \ldots, N, m=1, \ldots, M,
$$

the $2 \mathrm{D}$ spectral analysis problem of (25) converts to a conventional $1 \mathrm{D}$ problem. The observed sequence can therefore be written as

$$
\grave{\mathbf{y}}=\sum_{\zeta=1}^{M N} \sigma_{\zeta} \mathbf{a}_{\zeta}+\mathbf{n}
$$

And a reshaping operation of the $1 \mathrm{D}$ spectral estimation result $\sigma_{\zeta}$ outputs the final range-azimuth spectrum $\sigma_{n, m}$.

The WLS fitting criterion used by IAA is

$$
\widehat{\sigma}_{\zeta}=\arg \min _{\sigma_{\zeta}}\left\|\dot{\mathbf{y}}-\sigma_{\zeta} \mathbf{a}_{\zeta}\right\|_{\mathbf{R}_{i+n, \zeta}^{-1}}^{2}, \quad \zeta=1, \ldots, M N,
$$

in which $\mathbf{R}_{i+n, \zeta}$ is the interferences (signals at grid points other than the current one $\zeta$ ) and noise covariance matrix and square of the weighted $\ell_{2}$-norm are defined as $\|\mathbf{x}\|_{\mathbf{W}}^{2} \triangleq \mathbf{x}^{H} \mathbf{W} \mathbf{x}$.

The iterative solution to the weighted least-square problem (40) leads to the IAA algorithm. The solution to (40) is

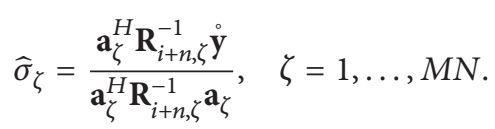

The interferences and noise covariance matrix can be written as

$$
\mathbf{R}_{i+n, \zeta}=\mathbf{R}-\left|\sigma_{\zeta}\right|^{2} \mathbf{a}_{\zeta} \mathbf{a}_{\zeta}^{H},
$$

where

$$
\mathbf{R}=E\left(\stackrel{\operatorname{yy}}{\circ}^{H}\right)=\sum_{\zeta=1}^{M N}\left|\sigma_{\zeta}\right|^{2} \mathbf{a}_{\zeta} \mathbf{a}_{\zeta}^{H}+\left[\begin{array}{cccc}
\gamma_{1} & 0 & \cdots & 0 \\
0 & \gamma_{2} & \cdots & 0 \\
\vdots & \vdots & \ddots & \vdots \\
0 & \cdots & \cdots & \gamma_{N_{t} N_{r}}
\end{array}\right]
$$

$$
\triangleq \mathbf{A P A}^{H}
$$

in which $\gamma_{i}$ is the noise power corresponding to the $i$ th virtual array element and

$$
\mathbf{A}=\left[\mathbf{a}_{1}, \ldots, \mathbf{a}_{M N}, \mathbf{I}\right] \triangleq\left[\mathbf{a}_{1}, \ldots, \mathbf{a}_{M N+N_{t} N_{r}}\right]
$$

$$
\begin{aligned}
& \text { initialize } \\
& i=0, \sigma_{\zeta}(0)=\frac{\mathbf{a}_{\zeta}^{H} \dot{\mathbf{y}}}{\left\|\mathbf{a}_{\zeta}\right\|^{2}}, \zeta=1, \ldots, M N+N_{t} N_{r} \\
& \text { repeat } \\
& \mathbf{R}(i)=\mathbf{A} \mathbf{P}(i) \mathbf{A}^{H} \\
& \sigma_{\zeta}(i+1)=\frac{\mathbf{a}_{\zeta}^{H} \mathbf{R}^{-1}(i) \stackrel{\circ}{\mathbf{y}}}{\mathbf{a}_{\zeta}^{H} \mathbf{R}^{-1}(i) \mathbf{a}_{\zeta}}, \zeta=1, \ldots, M N+N_{t} N_{r} \\
& i \longleftarrow i+1 \\
& \text { until (convergence) } \\
& \text { output } \\
& \sigma_{\zeta}(i), \zeta=1, \ldots, M N
\end{aligned}
$$

Algorithm 1: 2D IAA algorithm for the deramping based rangeazimuth processing.

is a $\left(N_{t} N_{r}\right) \times\left(M N+N_{t} N_{r}\right)$ matrix including all the steering vectors at different scanning grid points and also unitary vectors representing the noise components. The power term in (43) is

$$
\begin{aligned}
& \mathbf{P} \\
& =\left[\begin{array}{cc}
\operatorname{diag}\left(\left|\sigma_{1}\right|^{2}, \ldots,\left|\sigma_{M N}\right|^{2}\right) & 0 \\
\mathbf{0} & \operatorname{diag}\left(\left|\gamma_{1}\right|^{2}, \ldots,\left|\gamma_{N_{t} N_{r}}\right|^{2}\right)
\end{array}\right] \\
& \triangleq \operatorname{diag}\left(\left|\sigma_{1}\right|^{2}, \ldots,\left|\sigma_{M N}\right|^{2},\left|\sigma_{M N+1}\right|^{2}, \ldots,\left|\sigma_{M N+N_{t} N_{r}}\right|^{2}\right) \\
& \triangleq \operatorname{diag}\left(p_{1}, \ldots, p_{M N+N_{t} N_{r}}\right),
\end{aligned}
$$

which includes the power of the signal and noise.

Then using the matrix inversion lemma, the solution to the WLS problem indicated by (41) is equivalent to

$$
\widehat{\sigma}_{\zeta}=\frac{\mathbf{a}_{\zeta}^{H} \mathbf{R}^{-1} \mathbf{y}}{\mathbf{a}_{\zeta}^{H} \mathbf{R}^{-1} \mathbf{a}_{\zeta}}, \quad \zeta=1, \ldots, M N
$$

This avoids the computation of $\mathbf{R}_{i+n, \zeta}^{-1}$ for each scanning point, that is, $M N$ times. Moreover, $\left\{\widehat{\sigma}_{\zeta}\right\}$ can be computed in parallel for each scanning point, which makes IAA suitable to implement on parallel hardware.

Since the covariance matrix $\mathbf{R}$ depends on $\sigma_{\zeta}$ in turn, $\sigma_{\zeta}$ and $\mathbf{R}$ are estimated in an iterative manner, which yields the IAA algorithm shown in Algorithm 1.

Note that the noise component has to be included in the iteration to avoid the badly conditioned problem when calculating matrix inversion.

The convergence criterion is set to $\|\boldsymbol{\sigma}(i)-\boldsymbol{\sigma}(i-1)\|^{2} / \| \boldsymbol{\sigma}(i-$ 1) $\|^{2}<10^{-4}$ where $\sigma(i) \in \mathbb{C}^{M N \times 1}$ includes the estimate values at all the grid points in $i$ th iteration. However IAA usually does not provide significant improvements in performance after about 15 iterations. So we usually set the upper limit of iteration times to 10 20.

In each IAA iteration, the complexity (complex multiplication) of calculating covariance matrix $\mathbf{R}$ is $N_{t} N_{r}(M N+$ $\left.N_{t} N_{r}\right)^{2}+\left(N_{t} N_{r}\right)^{2}\left(M N+N_{t} N_{r}\right)$, and the complexity of 


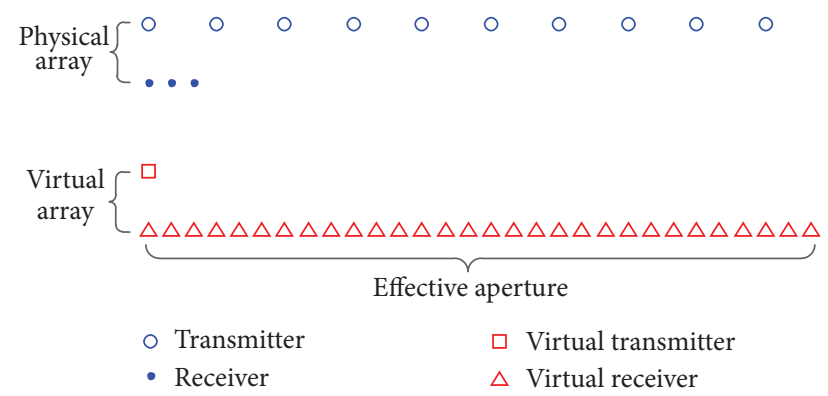

FIgURE 5: MIMO virtual array example.

calculating the inversion of covariance matrix is $\left(N_{t} N_{r}\right)^{3}$. Lastly, the range-azimuth spectrum at each grid point has to be estimated in each iteration, and its complexity is $2(M N+$ $\left.N_{t} N_{r}\right) \cdot\left(\left(N_{t} N_{r}\right)^{2}+N_{t} N_{r}\right)$. Assuming that the iteration times is a constant $W$, then the total computational complexity of the 2D IAA algorithm for the deramping based rangeazimuth spectrum estimation is $W\left[N_{t} N_{r}\left(M N+N_{t} N_{r}\right)^{2}+\right.$ $\left.\left(N_{t} N_{r}\right)^{2}\left(M N+N_{t} N_{r}\right)+2\left(M N+N_{t} N_{r}\right)\left(N_{t} N_{r}+1\right) N_{t} N_{r}\right]$, which is on the order of $\mathcal{O}\left(\left(M N+N_{t} N_{r}\right)^{2} N_{t} N_{r}\right)$. Thus the computational complexity heavily depends on the number of grid points in the range and azimuth angle domain.

\section{Design Example}

In this section, a complete design example of a MIMO sparse frequency FMCW radar is presented to demonstrate the proposed signal processing framework. The effectiveness of the modified 2D IAA algorithm for range-azimuth processing is also verified.

Consider a system with $N_{t}=10$ transmitting antennas and $N_{r}=3$ receiving antennas. And the antennas are arranged to form a ULA, with $d_{r}=0.5 \lambda$ and $d_{t}=N_{r} d_{r}=$ 1.5 $\lambda$. As shown in Figure 5, by arranging the antennas in this fashion, a filled virtual array with $N_{t} N_{r}=30$ antennas is created for higher angle resolution. The beamwidth of the virtual array is

$$
\Delta \theta=\frac{50.8 \lambda}{N_{t} N_{r} d_{r}}=3.4^{\circ}
$$

At the transmitter, a set of FMCW signals with identical parameters except the carrier frequency is broadcasted. The carrier frequencies are chosen according to Canada 1999 (summer) HF band spectrum monitoring result [11]. The clear channels are shown in Table 1 . The frequency range of 5.11 5.14 in Table 1 means that the radio spectrum upper than $5.11 \mathrm{MHz}$ and lower than $5.15 \mathrm{MHz}$ is clean. Thus the bandwidth of this band is $40 \mathrm{KHz}$. The start frequencies of the 10 transmit channels are accordingly chosen:

$$
\begin{aligned}
& f_{k} \\
& \in\{5.11,5.26,5.29,5.37,5.46,5.51,5.54,5.64,5.72,5.77\} \mathrm{MHz} .
\end{aligned}
$$

The minimum bandwidth of these channels is $20 \mathrm{KHz}$. Hence the bandwidth $B$ of the FMCW signal is set to $20 \mathrm{KHz}$ and the sweep period $T_{m}$ is set to $0.128 \mathrm{~s}$.
TABLE 1: Canada 1999 summer available spectrum [11].

\begin{tabular}{lcc}
\hline Channel & Frequency range $/ \mathrm{MHz}$ & Bandwidth $/ \mathrm{KHz}$ \\
\hline 1 & $5.11 \sim 5.14$ & 40 \\
2 & $5.26 \sim 5.27$ & 20 \\
3 & $5.29 \sim 5.31$ & 30 \\
4 & $5.37 \sim 5.39$ & 30 \\
5 & $5.46 \sim 5.48$ & 30 \\
6 & $5.51 \sim 5.52$ & 20 \\
7 & $5.54 \sim 5.59$ & 60 \\
8 & $5.64 \sim 5.66$ & 30 \\
9 & $5.72 \sim 5.73$ & 20 \\
10 & $5.77 \sim 5.80$ & 40 \\
\hline
\end{tabular}

The time-frequency characteristic of the transmitted signals of the whole array is shown in Figure 6. It also shows the two allocation strategies of the carrier frequencies. In Figure 6(a) the carrier frequencies are sequentially distributed while in Figure 6(b) the distribution of the carrier frequencies is optimized according to the criterion proposed at the end of Section 4 . The frequency sparsity of the transmitted signals is evident from Figure 6.

The ambiguity functions (periodogram range-azimuth images of a single target) of the transmit waveforms when using sequential carrier frequency distribution and optimized carrier frequency distribution are shown in Figures 7(a) and 7(b), respectively. In Figures 7(a) and 7(b), the range and azimuth plane cut at the peak location are placed below and left to the range-azimuth intensity plot. And Figure 7(c) shows the $-3 \mathrm{~dB}$ isolines of the ambiguity functions in both situations. The range-azimuth coupling is obvious when using sequential carrier frequency distribution as there exists a clear skewed ridge in the range-azimuth plot. And the ambiguity plot is distributed symmetrically relative to range and azimuth axes after the carrier frequency optimizing. Thus it can be concluded that the optimization of the carrier frequency distribution properly decouples the range and the azimuth angle estimate. Thus the optimized carrier frequency distribution shown in Figure 6(b) is used throughout this design example.

The frequency span of all the transmitted signals $(\Delta F)$ is $680 \mathrm{KHz}$, which corresponds to a fine measure resolution of $220.6 \mathrm{~m}$. The minimum frequency interval $\delta f=20 \mathrm{KHz}$ is larger than the frequency component corresponding to the maximum potential range of $300 \mathrm{~km}$, which is $2 \mu R_{\max } / c=$ $312.5 \mathrm{~Hz}$. Thus the condition of aliasing-free separation of the transmitted signals is satisfied.

In the simulation, we consider four targets whose information is shown in Table 2. These four targets are closely spaced in both range and azimuth domain. And their velocities are the same. Apparently, they fall in the same coarse range-velocity cell. The input SNRs of the targets are all set to be $-15 \mathrm{~dB}$. The number of sweep periods in a CPI is set to be 1024, corresponding to an integration time of 131 seconds and an integration gain of about $30 \mathrm{~dB}$.

After different transmitting channels are separated at each receiving channel, two sets of DFTs are applied to give 


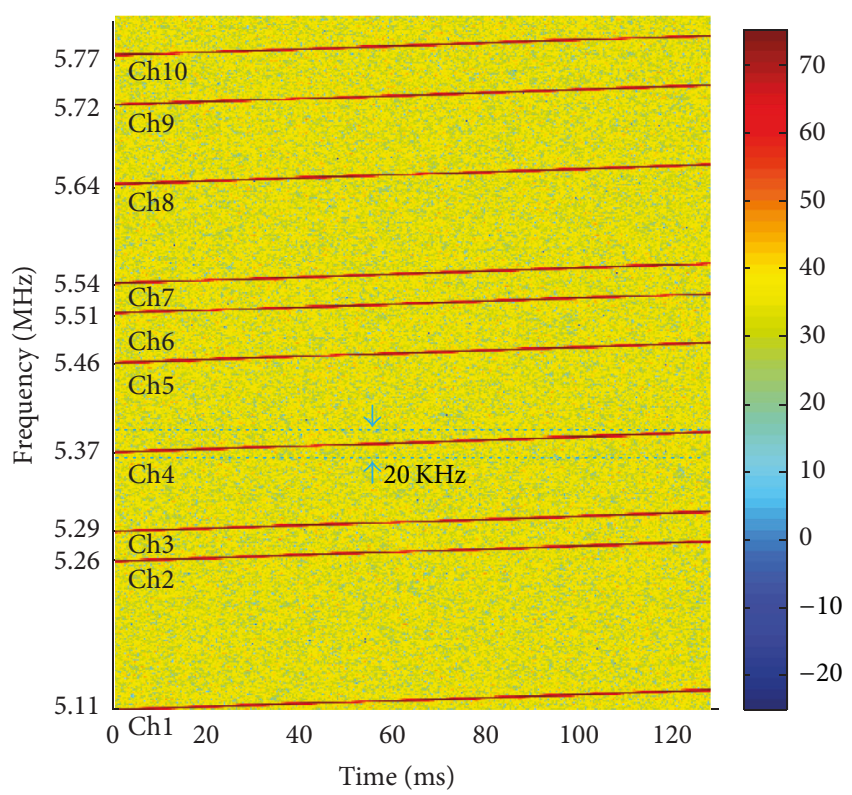

(a) Sequential distribution

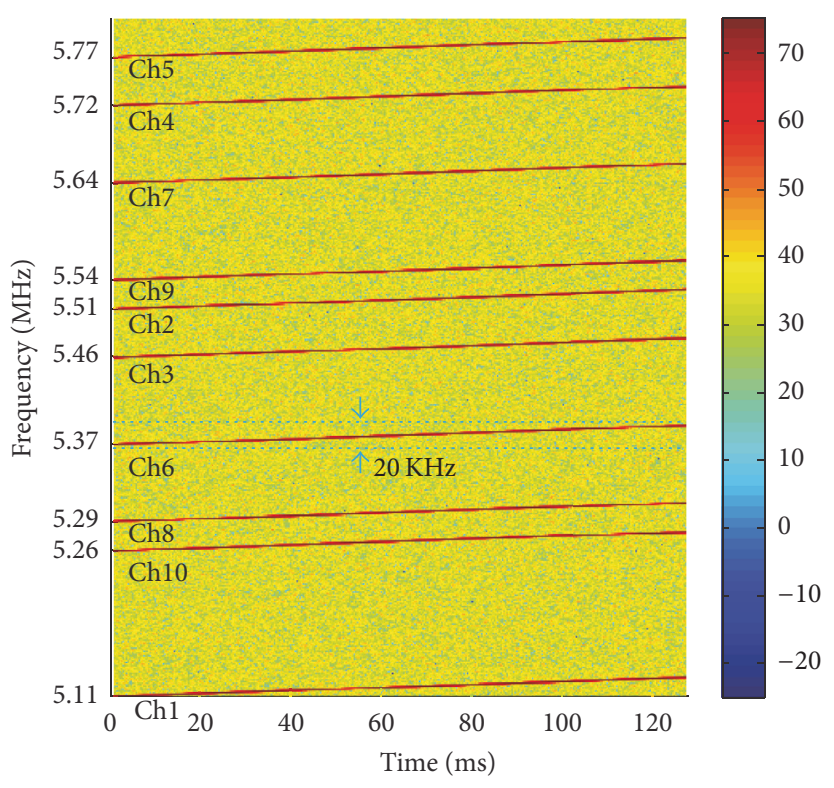

(b) Optimized distribution

FIGURE 6: Time-frequency characteristic of the transmitted signals (short-time Fourier transform (STFT) applied to the transmitted signals).

TABLE 2: Simulated target information.

\begin{tabular}{lcccc}
\hline Index & Range $/ \mathrm{km}$ & Azimuth $^{\circ}$ & Velocity $/ \mathrm{km} / \mathrm{h}$ & $\mathrm{SNR} / \mathrm{dB}$ \\
\hline 1 & 90 & 0 & 40 & -15 \\
2 & 90.2 & 3 & 40 & -15 \\
3 & 90 & 3 & 40 & -15 \\
4 & 90 & 0 & 40 & -15 \\
\hline
\end{tabular}

a range-velocity image in each $(l, k)$ channel. A Hamming window function is used for both the range domain DFT and the velocity domain DFT to lower the sidelobe levels in the range-velocity image. A waterfall plot of the range-velocity images of the first 4 channels is shown in Figure 8 . The first 4 channels have $l$ index equal to 0 and $k$ indices equal to $0,1,2,3$, respectively. In Figure 8, amplitudes of each channel are added by a specific value to produce a "waterfall" effect. In Figure 8(a), range and velocity profiles at the peak location are plotted using black lines for each range-velocity image. They are also listed in Figures 8(b) and 8(c) for clarity. Coarse range and velocity information of the targets can be determined from the range-velocity images. True values of target range and velocity have been marked by the red dotted lines. The four targets can not be separated using a single channel; that is, they are not separable before range-azimuth processing (synthetic impulse and aperture processing).

In this simulation experiment, peak values of the $N_{t} N_{r}$ channels are fed into the range-azimuth processor to further determine the target range and target azimuth angle. However, in reality, range-azimuth processing may be applied in a larger range window which includes several coarse range cell and the fine measure results are spliced to form a complete range-azimuth image.
The searching regions in the range and azimuth domain are set to $R=\{r \mid 89 \mathrm{~km} \leq r \leq 91 \mathrm{~km}\}$ and $\Theta=\left\{\theta \mid-30^{\circ} \leq\right.$ $\left.\theta \leq 30^{\circ}\right\}$, respectively. In this paper, we have introduced the conventional periodogram approach and proposed a modified deramping processing based $2 \mathrm{D}$ iterative adaptive approach (IAA) for the range-azimuth processing. Their results are shown in Figure 9. We have marked black circles at the true positions of the four targets in the range-azimuth plane. From the range-azimuth results, we can observe that periodogram approach almost fails due to the sparsity of the transmitting carrier frequencies. The IAA algorithm results in a quite clear range-azimuth image with the four targets properly separated and the sidelobes kept at an acceptable level. Figure 10 shows the range and azimuth profiles of the IAA range-azimuth image at the target positions. The closely placed targets are properly resolved and the peak locations of the spectrum provide a fine estimate for the target range and azimuth.

Detection can be processed on the range-azimuth plane as shown in Figure 11(a). The most commonly used cell averageconstant false alarm rate (CA-CFAR) detector is used for demonstration. And the protection cells and reference cells are chosen from the cells located in the rectangle centered at the detection cell. The detection threshold and result on the IAA range-azimuth spectrum are shown in Figure 11(b) at a detection factor of $13 \mathrm{~dB}$. The upper surface plot in cool color scheme is the CFAR threshold while the lower surface plot in hot color scheme is the CFAR input (rangeazimuth spectrum). The blue solid points mark the points that are above the threshold and the black circles mark the true locations of the four targets. Thus we can observe that cohesion of the detection results can properly output the target information for the subsequent data processor and display terminal. 


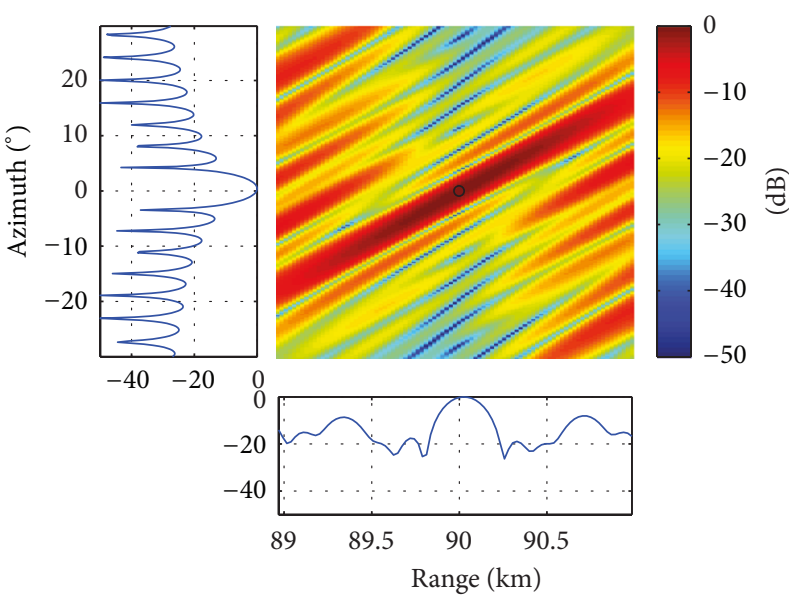

(a) Sequential distribution

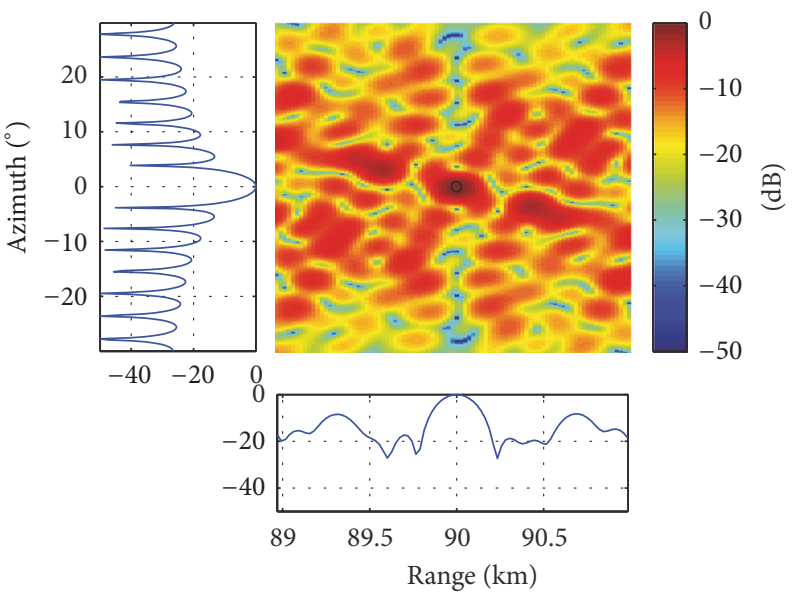

(b) Optimized distribution

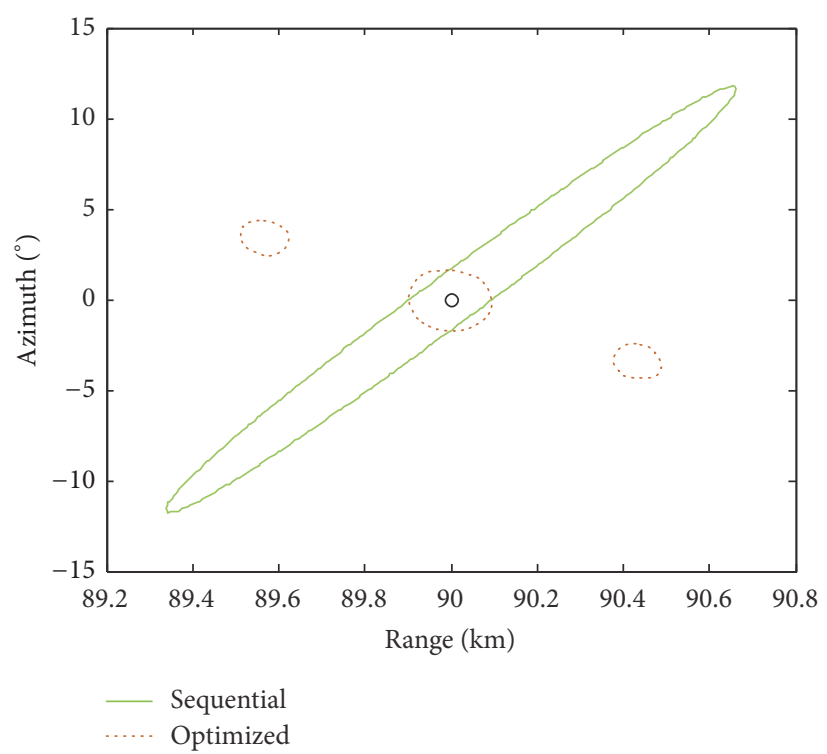

(c) Plots of the $-3 \mathrm{~dB}$ isolines

Figure 7: Ambiguity functions of the transmit waveforms when using sequential carrier frequency distribution and optimized carrier frequency distribution.

\section{Conclusion}

The highly congested radio spectrum environment in HF band seriously limits the range resolution of HFSWR. MIMO sparse frequency FMCW radar is a solution to synthesizing wideband waveform in this situation by utilizing spatial frequency diversity. It is especially suitable for the HFSWR in many other aspects.

In this paper, we proposed a deramping based processing framework for the MIMO sparse frequency FMCW radar. Echo model of nonstationary target is formulated and all the intermediate results of the processing flow are presented. Several parameter selection criteria are also discussed in this paper. After deramping and channel separating, routine of range-velocity is followed in each channel to improve target SNR and separate targets by their coarse ranges and velocities. Actually, due to the high Doppler resolution of HFSWR, most targets or targets and components of sea backscatter can be separated by their velocities. Targets' coarse ranges and velocities are obtained by the peak locations in the rangevelocity images.

Values of the peak locations at different channels are fed into the range-azimuth processor which is actually a $2 \mathrm{D}$ spectral estimator to further determine the target azimuth angles and resolve the targets falling in the same rangevelocity cell. Input signal of the range-azimuth processor was shown to be identical to that of the synthetic impulse and aperture processor (SIAP) of the SIAR system. The ambiguity function of the range-azimuth processing was derived and based on it, temporal and spatial resolution and the rangeazimuth coupling were analyzed. When sequential carrier frequency distribution is used for the transmitting signals, the coupling is severe which will cause a large estimation error. Rearranging the carrier frequencies over the transmitting 


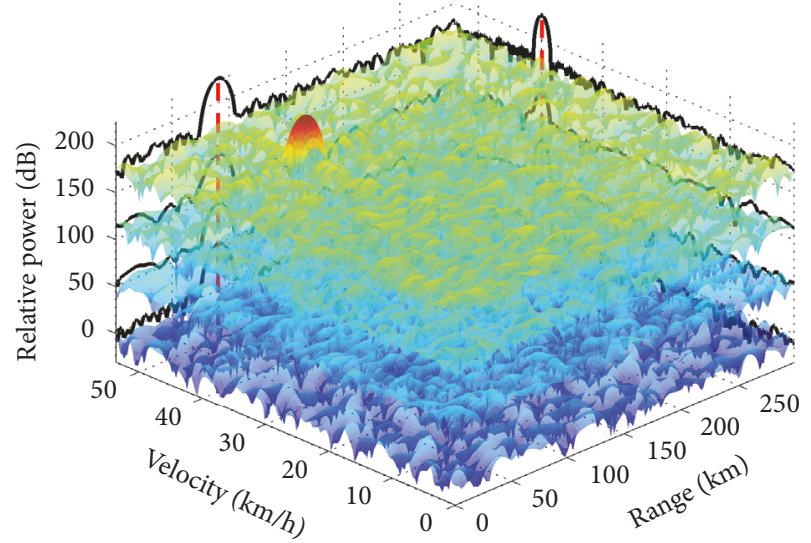

(a) 3D waterfall plot

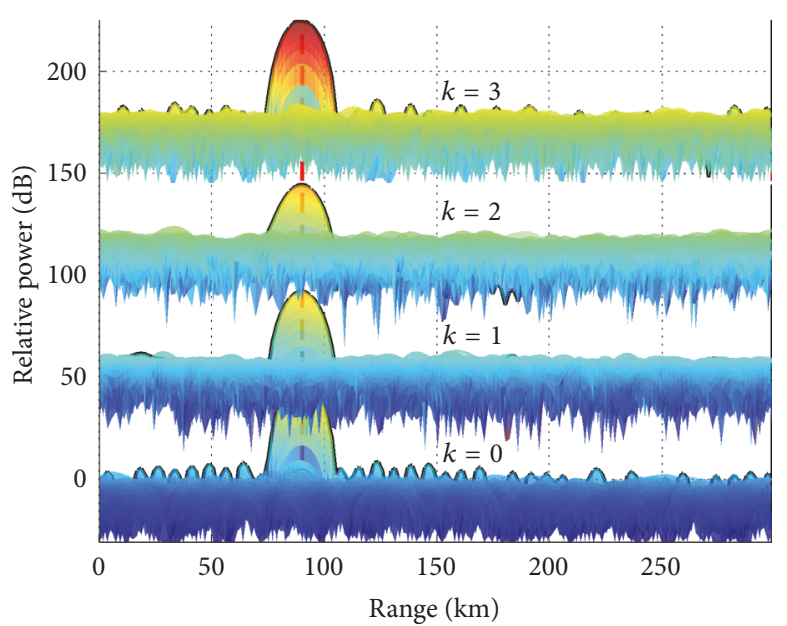

(b) Range profile

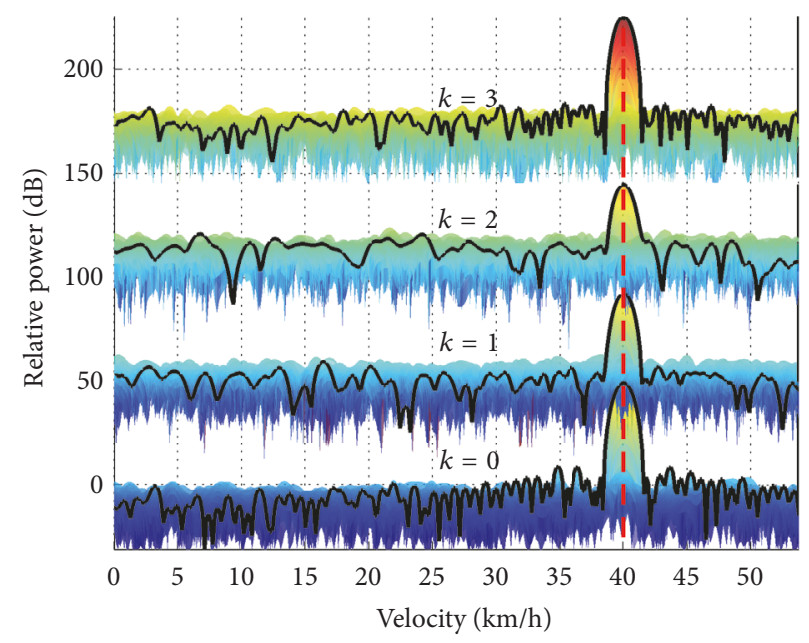

(c) Velocity profile

FIGURE 8: Waterfall plot of the range-velocity images of different channels.

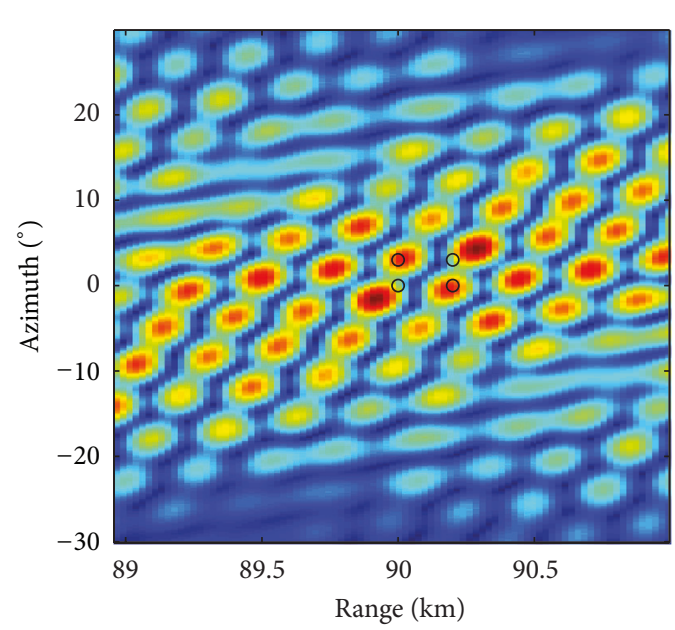

(a) Result of periodogram

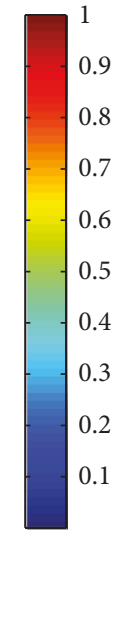

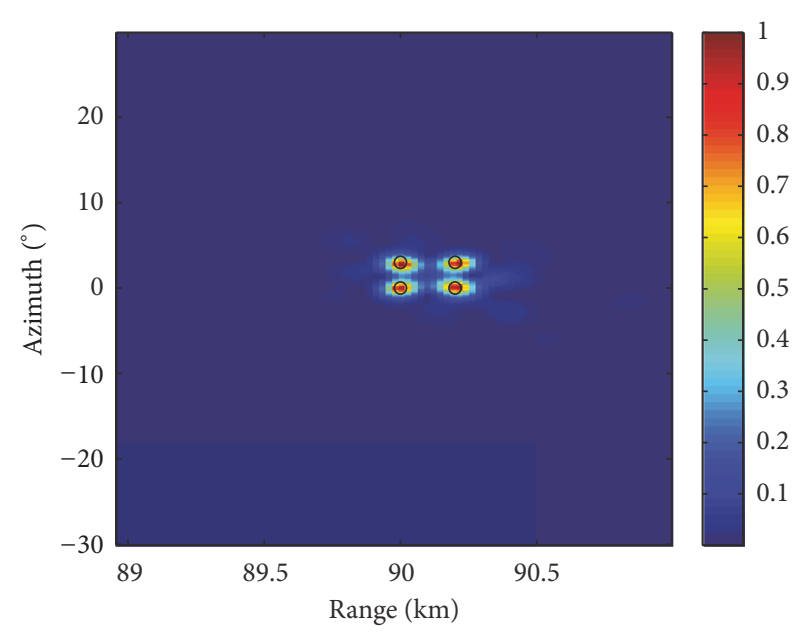

(b) Result of modified 2D IAA

FIGURE 9: Range-azimuth image formed by periodogram and modified 2D IAA. 

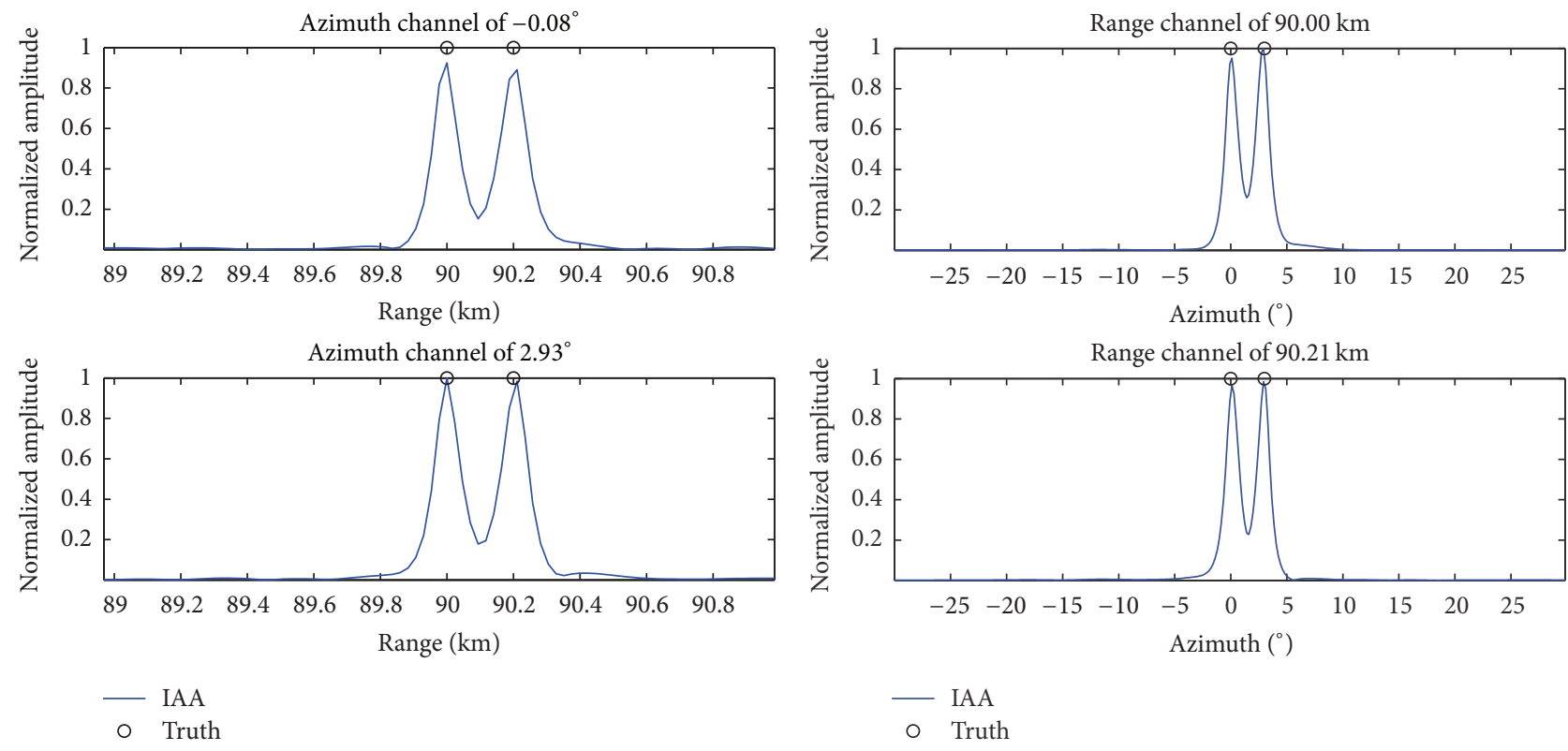

(a) Range profile

(b) Azimuth profile

FIGURE 10: Range and azimuth profile of 2D IAA range-azimuth image at the target positions.

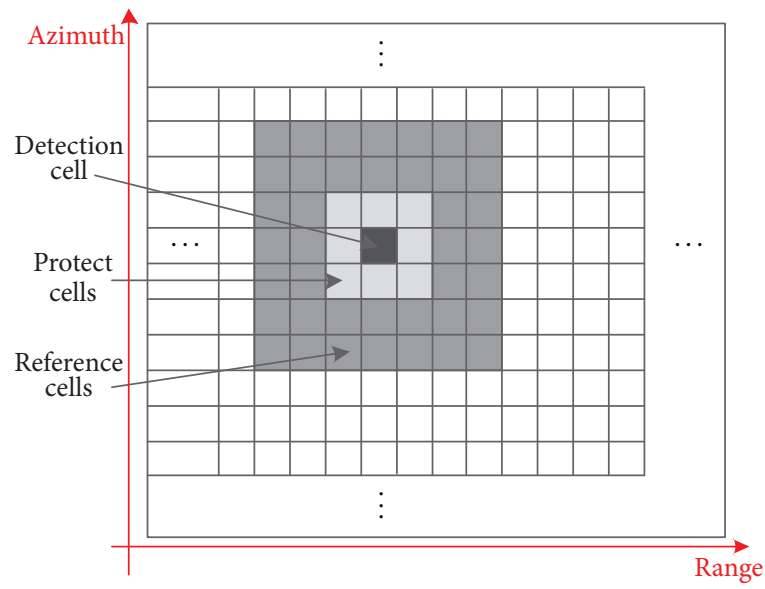

(a) Two-dimensional CFAR detection illustration

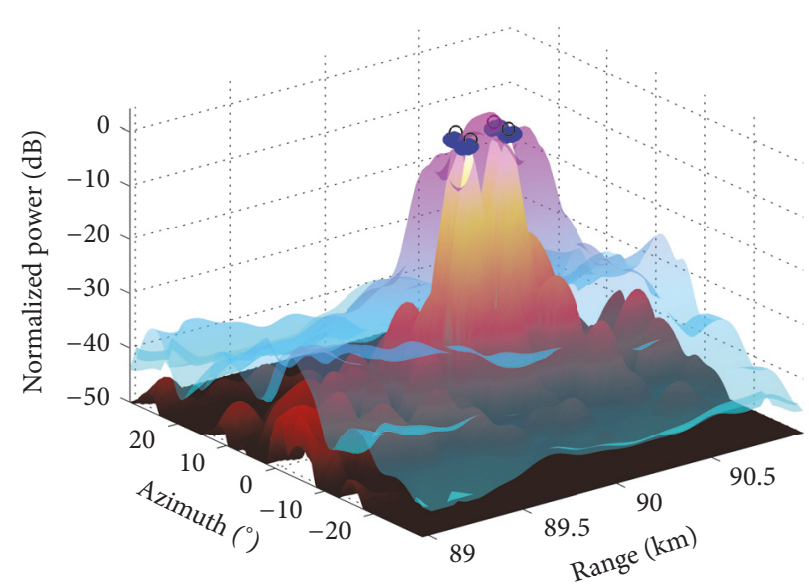

(b) Detection results on 2D IAA range-azimuth spectrum

FIGURE 11: The detection process demonstration.

sensors is a simple and effective decoupling solution. The process of finding the best arrangement strategy has been modeled as a combinatorial optimization problem in this paper.

The sparsity of the carrier frequencies makes $2 \mathrm{D}$ periodogram method fail when it is applied in the range-azimuth processor. Based on common 1D IAA, we proposed a concise 2D IAA method which is especially compatible with our deramping based model. The computational complexity of modified 2D IAA was also analyzed in this paper.

Finally, a complete design example demonstrated the whole proposed processing flow and validated the effectiveness of modified $2 \mathrm{D}$ IAA when using in the range-azimuth processing.

\section{Conflicts of Interest}

The authors declare that there are no conflicts of interest regarding the publication of this paper.

\section{References}

[1] L. Sevgi, A. Ponsford, and H. C. Chan, "An integrated maritime surveillance system based on high-frequency surfacewave radars, Part 1. Theoretical background and numerical simulations," IEEE Antennas and Propagation Magazine, vol. 43, no. 4, pp. 28-43, 2001.

[2] A. M. Ponsford, L. Sevgi, and H. C. Chan, "An integrated maritime surveillance system based on high-frequency surfacewave radar, part II: operational status and system performance," 
IEEE Antennas and Propagation Magazine, vol. 43, no. 5, pp. 5263, 2001.

[3] H. J. Roarty, D. E. Barrick, J. T. Kohut, and S. M. Glenn, “Dualuse of compact HF radars for the detection of midand large-size vessels," Turkish Journal of Electrical Engineering \& Computer Sciences, vol. 18, no. 3, pp. 373-388, 2010.

[4] A. M. Ponsford and J. Wang, "A review of high frequency surface wave radar for detection and tracking of ships," Turkish Journal of Electrical Engineering and Computer Sciences, vol. 18, no. 3, pp. 409-428, 2010.

[5] D. E. Barrick, "HF radio oceanography-a review," BoundaryLayer Meteorology, vol. 13, no. 1-4, pp. 23-43, 1978.

[6] D. E. Barrick, J. M. Headrick, R. W. Bogle, and D. D. Crombie, "Sea backscatter at HF: interpretation and utilization of the echo," Proceedings of the IEEE, vol. 62, no. 6, pp. 673-680, 1974.

[7] D. E. Barrick, M. W. Evans, and B. L. Weber, "Ocean surface currents mapped by radar," Science, vol. 198, no. 4313, pp. 138144, 1977.

[8] K. Hasselmann, "Determination of ocean wave spectra from doppler radio return from the sea surface," Nature, vol. 229, no. 1, pp. 16-17, 1971.

[9] D. Barrick, "History, present status, and future directions of HF surface-wave radars in the U.S," in Proceedings of the International Conference on Radar (RADAR '03), pp. 652-655, Adelaide, Australia, September 2003.

[10] K.-W. Gurgel, G. Antonischki, H.-H. Essen, and T. Schlick, "Wellen radar (WERA): a new ground-wave hf radar for ocean remote sensing," Coastal Engineering, vol. 37, no. 3-4, pp. 219234, 1999.

[11] W. H. L. Hank and D. Barry, "Channel availability for east coast high frequency surface wave radar systems," Tech. Rep., Canada, Defence R\&D, 2001.

[12] A. Dzvonkovskaya and H. Rohling, "Software-improved range resolution for oceanographic HF FMCW radar," in Proceedings of the 14th International Radar Symposium (IRS '13), pp. 411-416, June 2013.

[13] V. M. Kutuzov, "Synthesis of non-regular multitone signals and algorithms of their processing," in Proceedings of the $3 \mathrm{rd}$ International Conference on Signal Processing (ICSP '96), vol. 1, pp. 813-816, October 1996.

[14] S. Green and S. P. Kingsley, "Improving the range/time sidelobes of large bandwidth discontinuous spectra HF radar waveforms," in Proceedings of the 7th International Conference on High Frequency Radio Systems and Techniques (Conf. Publ. No. 441), pp. 246-250, IEEE, Nottingham, UK, July 1997.

[15] S. D. Green and S. P. Kingsley, "Investigation of wide bandwidth HF radar waveforms," in Proceedings of the IEE Electronics Division Colloquium on Advanced Transmission Waveforms, pp. 2/1-2/8, June 1995.

[16] M. J. Lindenfeld, "Sparse frequency transmit and receive waveform design," IEEE Transactions on Aerospace and Electronic Systems, vol. 40, no. 3, pp. 851-861, 2004.

[17] G. H. Wang and Y. L. Lu, "Designing single/multiple sparse frequency waveforms with sidelobe constraint," IET Radar, Sonar and Navigation, vol. 5, no. 1, pp. 32-38, 2011.

[18] M. Pan, B. Chen, and M. Yang, "A general range-velocity processing scheme for discontinuous spectrum FMCW signal in HFSWR applications," International Journal of Antennas and Propagation, vol. 2016, Article ID 2609873, 13 pages, 2016.

[19] D. E. Barrick, "FM/CW radar signals and digital processing," Tech. Rep., National Oceanic and Atmospheric Administration, Boulder, CO, USA, 1973.
[20] H. C. Chan, "Evaluation of the FMICW waveform in HF surface radar applications," Tech. Rep., Defence Research Establishment, Ottawa, Canada, 1994.

[21] A.-S. Luce, H. Molina, D. Muller, and V. Thirard, "Experimental results on RIAS digital beamforming radar," in Proceedings of the International Conference (Radar '92), pp. 74-77, October 1992.

[22] C. Baixiao and W. Jianqi, Synthetic Impulse and Aperture Radar (SIAR): A Novel Multi-Frequency MIMO Radar, John Wiley \& Sons, 2014.

[23] D. Chen, B. Chen, and S. Zhang, "Multiple-input multipleoutput radar and sparse array synthetic impulse and aperture radar," in Proceedings of the CIE International Conference on Radar (ICR '06), pp. 1-4, Shanghai, China, October 2006.

[24] J. Li, P. Stoica, L. Xu, and W. Roberts, "On parameter identifiability of MIMO radar," IEEE Signal Processing Letters, vol. 14, no. 12, pp. 968-971, 2007.

[25] J. Li and P. Stoica, "MIMO radar with colocated antennas," IEEE Signal Processing Magazine, vol. 24, no. 5, pp. 106-114, 2007.

[26] D. W. Bliss and K. W. Forsythe, "Multiple-input multipleoutput (MIMO) radar and imaging: degrees of freedom and resolution," in Proceedings of the Conference Record of the 37th IEEE Asilomar Conference on Signals, Systems and Computers (ACSSC '03), vol. 1, pp. 54-59, Pacific Grove, Calif, USA, November 2003.

[27] G. J. Frazer, Y. I. Abramovich, and B. A. Johnson, "Spatially waveform diverse radar: perspectives for high frequency OTHR," in Proceedings of the IEEE 2007 Radar Conference, pp. 385-390, April 2007.

[28] M. Lesturgie, "Improvement of high-frequency surface waves radar performances by use of multiple-input multiple-output configurations," IET Radar, Sonar and Navigation, vol. 3, no. 1, pp. 49-61, 2009.

[29] G. Wang and Y. Lu, "High resolution MIMO-HFSWR using sparse frequency waveform," in Proceedings of the 2008 9th International Conference on Signal Processing (ICSP '08), pp. 2238-2241, October 2008.

[30] B. Chen, D. Chen, S. Zhang, H. Zhang, and M. Liu, "Experimental system and experimental results for coast-ship Bi/multistatic ground-wave over-the-horizon radar," in Proceedings of the 2006 CIE International Conference on Radar (ICR '06), pp. 1-5, October 2006.

[31] H. Su, H. Liu, P. Shui, S. Zhang, and Z. Bao, "HFSWR based on synthetic impulse and aperture processing," IEE Proceedings: Radar, Sonar and Navigation, vol. 153, no. 5, pp. 445-453, 2006.

[32] J. J. M. De Wit, W. L. Van Rossum, and A. J. De Jong, "Orthogonal waveforms for FMCW MIMO radar," in Proceedings of the 2011 IEEE Radar Conference: In the Eye of the Storm (RadarCon '11), pp. 686-691, May 2011.

[33] G. Babur, O. A. Krasnov, A. Yarovoy, and P. Aubry, "Nearly orthogonal waveforms for MIMO FMCW radar," IEEE Transactions on Aerospace and Electronic Systems, vol. 49, no. 3, pp. 1426-1437, 2013.

[34] T. Yardibi, J. Li, P. Stoica, M. Xue, and A. B. Baggeroer, "Source localization and sensing: a nonparametric iterative adaptive approach based on weighted least squares," IEEE Transactions on Aerospace and Electronic Systems, vol. 46, no. 1, pp. 425-443, 2010.

[35] P. Stoica, J. Li, and H. He, "Spectral analysis of nonuniformly sampled data: a new approach versus the periodogram," IEEE Transactions on Signal Processing, vol. 57, no. 3, pp. 843-858, 2009. 
[36] X. Tan, W. Roberts, J. Li, and P. Stoica, "Range-Doppler imaging via a train of probing pulses," IEEE Transactions on Signal Processing, vol. 57, no. 3, pp. 1084-1097, 2009.

[37] W. Roberts, P. Stoica, J. Li, T. Yardibi, and F. A. Sadjadi, "Iterative adaptive approaches to MIMO radar imaging," IEEE Journal on Selected Topics in Signal Processing, vol. 4, no. 1, pp. 5-20, 2010.

[38] Y. Liu, R. Xu, and N. Zhang, "Progress in HFSWR research at Harbin Institute of Technology," in Proceedings of the International Conference on Radar (RADAR '03), pp. 522-528, September 2003.

[39] D. J. Emery and G. Dickel, "The operation and performance of a multi-frequency HF Surfacewave radar," in Proceedings of the 2008 IEEE Radar Conference (RADAR '08), pp. 1-6, May 2008.

[40] A. L. Dzvonkovskaya, L. I. Dzvonkovsky, and V. A. Sobchuk, "Adaptive selection of HF radar operating channels," in Proceedings of the 2008 International Conference on Radar (Radar '08), pp. 230-234, September 2008.

[41] G. F. Earl and B. D. Ward, "The frequency management system of the Jindalee over-the-horizon backscatter HF radar," Radio Science, vol. 22, no. 2, pp. 275-291, 1987.

[42] International Telecommunication Union, "Recommendation ITU-R PI.372-8-Radio Noise," 2003. 


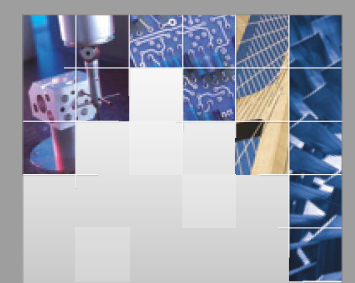

\section{Enfincering}
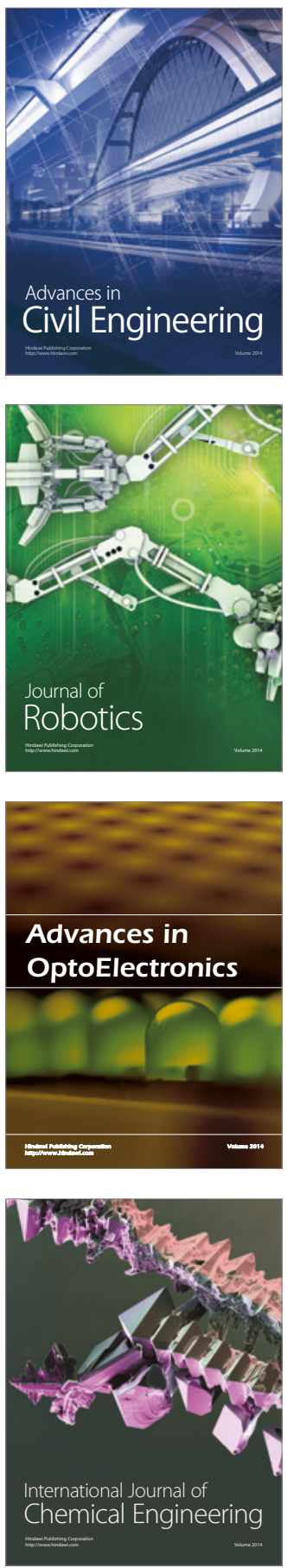

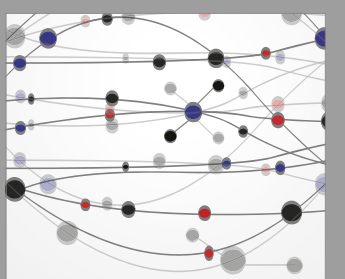

The Scientific World Journal

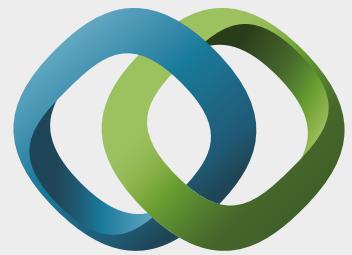

\section{Hindawi}

Submit your manuscripts at

https://www.hindawi.com
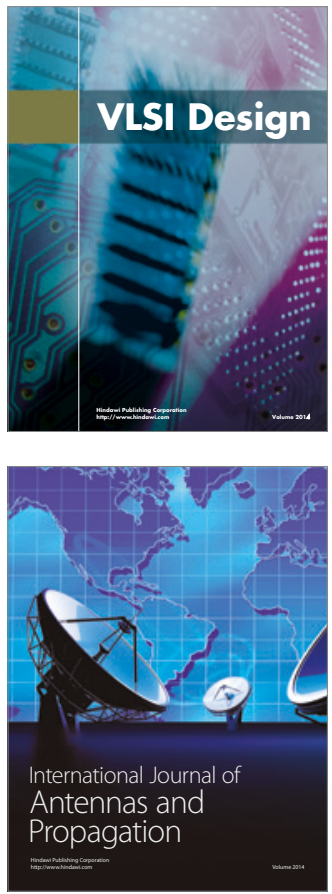

\section{Rotating}

Machinery
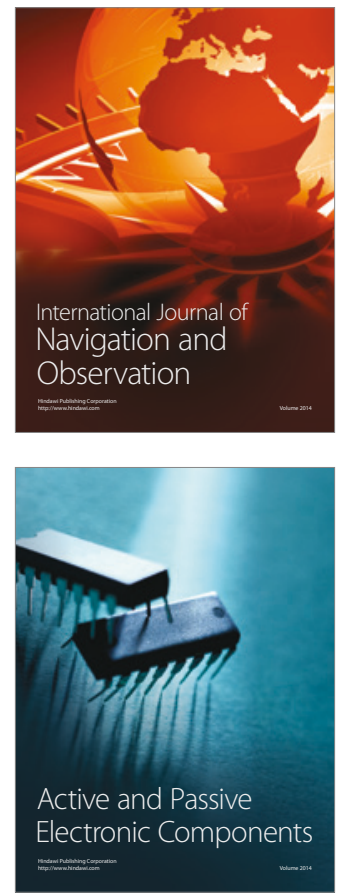
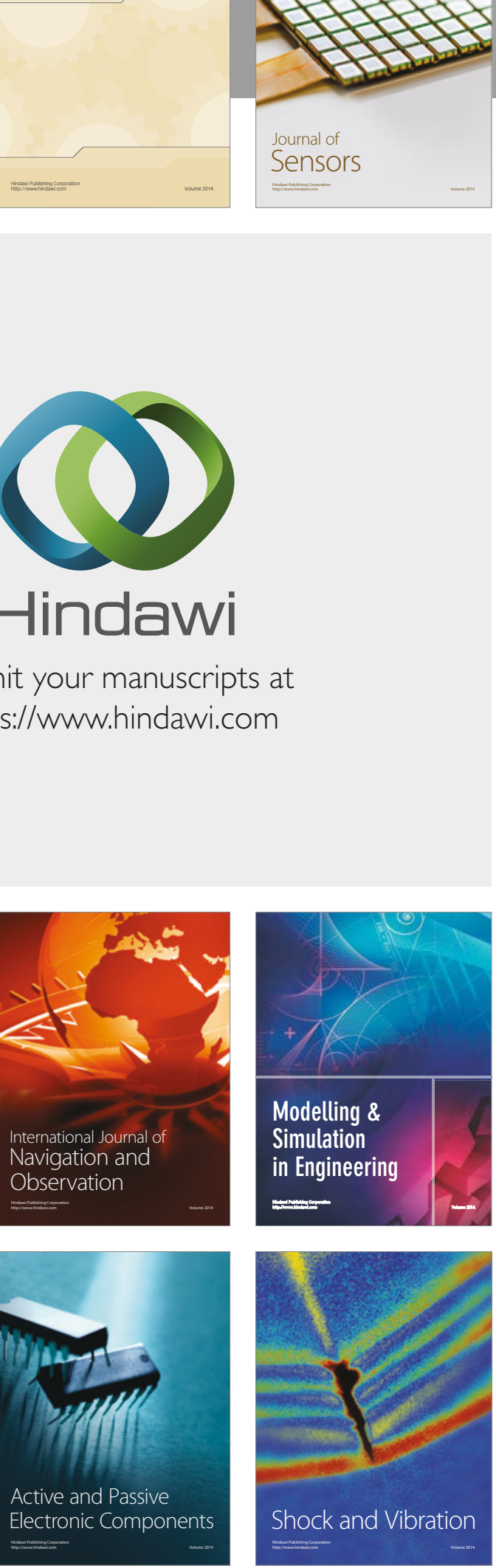
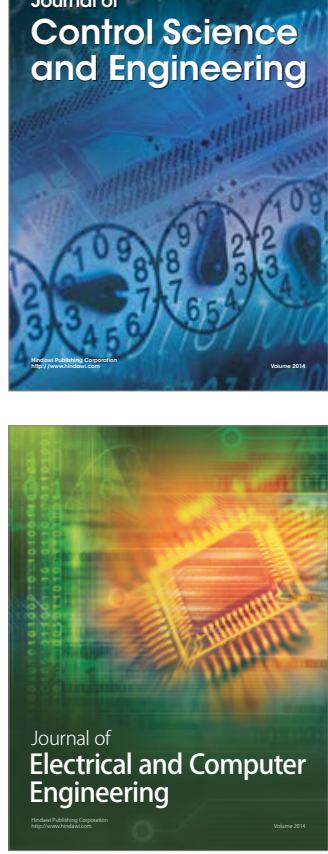

Distributed

Journal of

Control Science

and Engineering
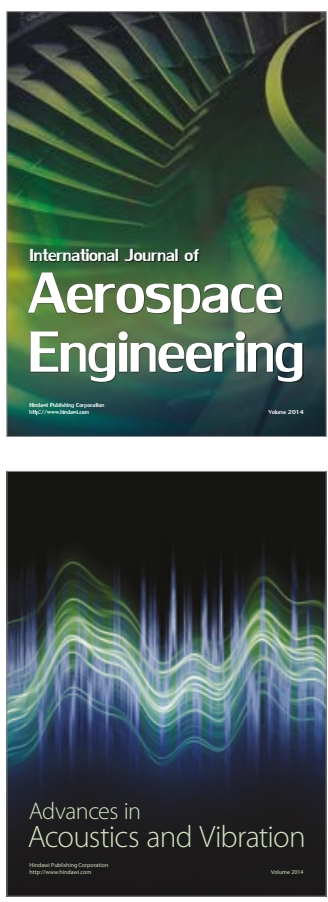

Sensor Networks 